ARTICLE

Received 13 May 2015 | Accepted 23 May 2017 | Published 27 Jul 2017

DOl: $10.1038 /$ ncomms 15990

OPEN

\title{
Regulation of Drosophila hematopoietic sites by Activin- $\beta$ from active sensory neurons
}

Kalpana Makhijani ${ }^{1}$, Brandy Alexander ${ }^{1}$, Deepti Rao ${ }^{1}$, Sophia Petraki ${ }^{1}$, Leire Herboso ${ }^{1}$, Katelyn Kukar ${ }^{1}$, Itrat Batool ${ }^{1}$, Stephanie Wachner ${ }^{1, \dagger}$, Katrina S. Gold ${ }^{1}$, Corinna Wong ${ }^{1}$, Michael B. O'Connor ${ }^{2}$ \& Katja Brückner ${ }^{1,3}$

An outstanding question in animal development, tissue homeostasis and disease is how cell populations adapt to sensory inputs. During Drosophila larval development, hematopoietic sites are in direct contact with sensory neuron clusters of the peripheral nervous system (PNS), and blood cells (hemocytes) require the PNS for their survival and recruitment to these microenvironments, known as Hematopoietic Pockets. Here we report that Activin- $\beta$, a TGF- $\beta$ family ligand, is expressed by sensory neurons of the PNS and regulates the proliferation and adhesion of hemocytes. These hemocyte responses depend on PNS activity, as shown by agonist treatment and transient silencing of sensory neurons. Activin- $\beta$ has a key role in this regulation, which is apparent from reporter expression and mutant analyses. This mechanism of local sensory neurons controlling blood cell adaptation invites evolutionary parallels with vertebrate hematopoietic progenitors and the independent myeloid system of tissue macrophages, whose regulation by local microenvironments remain undefined.

\footnotetext{
${ }^{1}$ Eli and Edythe Broad Center of Regeneration Medicine and Stem Cell Research, and Department of Cell and Tissue Biology, University of California San Francisco, San Francisco, California 94143, USA. ${ }^{2}$ Department of Genetics, Cell Biology and Development, University of Minnesota, Minneapolis, Minnesota 55455, USA. ${ }^{3}$ Cardiovascular Research Institute, and Helen Diller Family Comprehensive Cancer Center, University of California San Francisco, San Francisco, California 94143, USA. † Present address: Institute of Science and Technology (IST) Austria, 3400 Klosterneuburg, Austria. Correspondence and requests for materials should be addressed to K.B. (email: katja.brueckner@ucsf.edu).
} 
$\mathrm{n}$ vertebrates, regulation of self-renewing blood cell populations by local organ microenvironments is poorly understood at the cellular and molecular level ${ }^{1-3}$. In a related Drosophila melanogaster model, blood cells (hemocytes), with similarities to vertebrate tissue macrophages and oligopotent hematopoietic progenitors, form resident clusters in segmentally repeated inductive microenvironments of the larval body wall, also known as Hematopoietic Pockets (HPs $)^{4-7}$ (Fig. 1a). More than $90 \%$ of these larval hemocytes of embryonic origin are macrophage-like cells (plasmatocytes), which in the larva colonize HPs and expand by proliferation in the differentiated state $^{4}$. Based on their functional dependence on sensory neurons of the HPs for their localization and survival, and the elevated proliferation of resident hemocytes compared to those in circulation $^{4}$, we investigated the molecular mechanism of hemocyte induction by the sensory neurons of the peripheral nervous system (PNS).

Here we identify a molecular mechanism by which Drosophila hematopoiesis is controlled by the PNS. Activin- $\beta$ (Act $\beta, A c t)$, a Transforming Growth Factor- $\beta$ (TGF- $\beta$ ) family ligand, is specifically produced by multidendritic neurons and chordotonal organs of the PNS, and acts to regulate the proliferation and longterm adhesion of hemocytes. PNS activation by agonist treatment drives expansion of the blood cell pool, while specific silencing of sensory neurons affects resident hemocyte number and localization. Act $\beta$ plays a key role in this regulation, as evidenced by the induction of $A c t \beta$ expression in response to PNS activity, and a blunted response in Act $\beta$ mutants. These findings shed new light on the mechanisms by which local microenvironments regulate blood cell adaptation and may integrate sensory inputs.

\section{Results}

Sensory neurons form an interface with the blood cell system. First we examined the local anatomy of PNS neurons and resident (sessile) hemocytes. High magnification imaging revealed that PNS neurons form intricate extensions to areas of resident hemocytes (Fig. 1b), suggesting an interface that allows direct neuron-to-hemocyte communication. Using a split GFP approach, GFP Reconstitution Across Synaptic Partners (GRASP) $^{8,9}$ (Supplementary Fig. 1a), we confirmed that hemocytes are anatomically extremely close, and potentially form direct contacts with PNS neurons and glia (Supplementary Fig. 1b-e). To identify specific molecular signals that mediate this communication, we screened components of several key signalling pathways utilizing in vivo RNA interference (RNAi) $^{10}$, searching for defects in hemocyte number and/or localization. Based on this, we focused on the role of the TGF- $\beta$ family-related dSmad2 (Smox) pathway ${ }^{11}$ in hemocyte regulation. To identify the responsible ligand, we examined expression of the putative pathway ligands Activin- $\beta$ (Act $\beta$ ), Dawdle (daw) and myoglianin (myo) using ligand GAL4 reporters (O'Connor lab and $\left.{ }^{2,13}\right)$. Act $\beta$ was highly expressed by specific subsets of sensory neurons in the HPs, in particular the multidendritic (md) neurons and chordotonal organs ${ }^{14}$ (Fig. 1d,e). None of the other ligands showed obvious expression in the sensory neurons. Colabelling confirmed localization of $A c t \beta$-producing neurons with resident hemocytes in the HPs (Fig. 1c). In contrast, Act $\beta$ was not detectably expressed in other components of the HPs, such as epidermis, muscle and oenocytes, (Figs 1c,d,6c,d,f,g). Act $\beta$ was also highly expressed in motor neurons and other neurons of the central nervous system (CNS) as described previously, similar to related ligands of the TGF- $\beta$ family ${ }^{11,15-20}$. However, the vast majority of hemocytes in the larva, present as resident hemocytes in the HPs, were physically separated from the CNS, motor

\section{a}

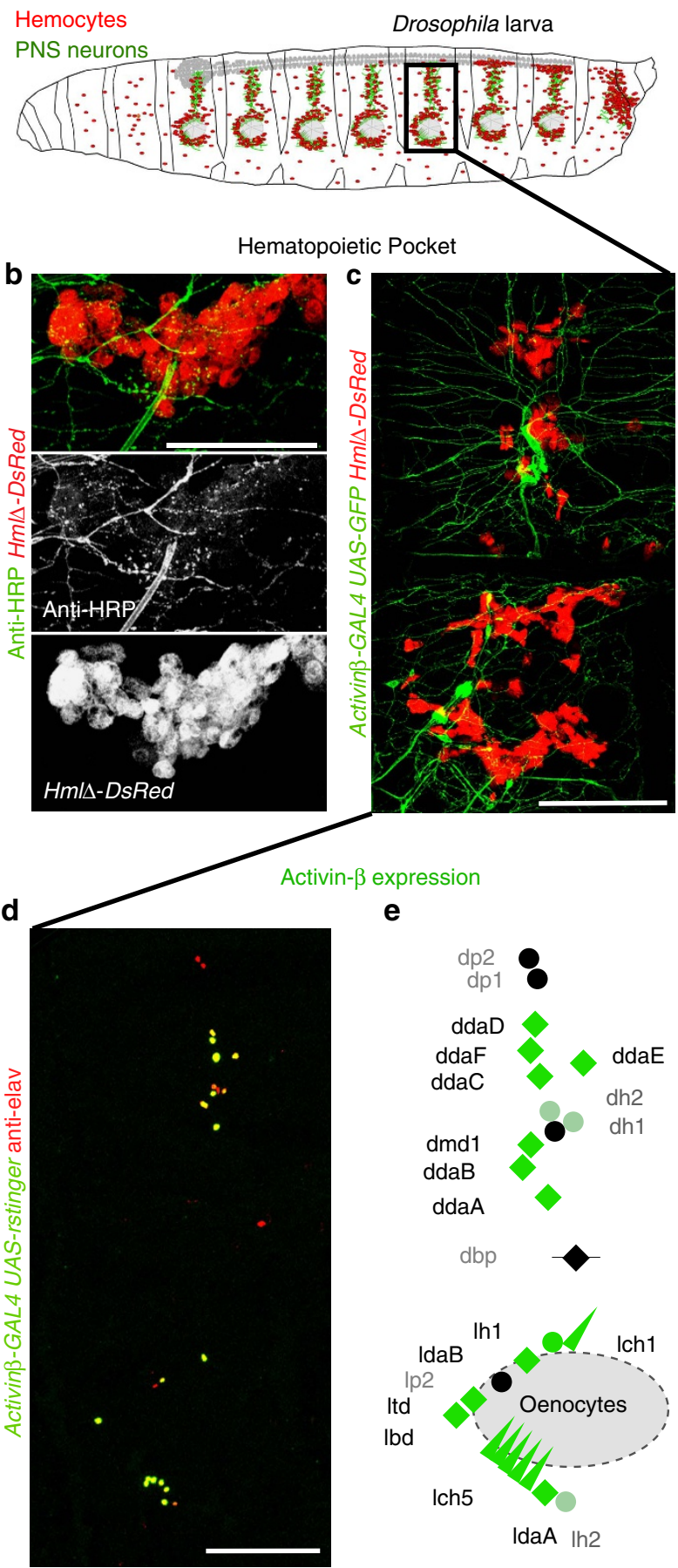

Figure 1 | PNS sensory neurons produce Activin- $\beta$ and are surrounded by hemocytes. (a) Model of a Drosophila larva; hemocytes in red, neurons in green. Boxed area marks a HP. (b) Close-up of a lower part of a HP illustrating intricate neuronal extensions in areas of hemocytes; neurons (Anti-HRP, green), hemocytes (Hml $\Delta$-DsRed, red), lateral patch area. Middle and lower panel show single channels. (c) Act $\beta$ expressing larval PNS neurons marked by reporter Act $\beta$-GAL4; UAS-mCD8GFP (green), Act $\beta$ positive neurons colocalize with hemocytes marked by $\mathrm{Hml} \Delta$-DsRed (red). Fillet prep containing all tissue layers. (d) Act $\beta$ expression pattern in larval PNS neurons, Act $\beta$ GAL4/ + ; UAS-r-stinger/ + (green), pan-neuronal anti-elav (red). Fillet prep containing all tissue layers. (e) Model of Act $\beta$ expression (green) in most multidendritic neurons (diamonds), chordotonal organs (triangles), and some external sensory neurons (circles). Scale bars, b, $50 \mu \mathrm{m} ; \mathbf{c}, \mathbf{d}$, $100 \mu \mathrm{m}$. 
neurons and axon terminals of motor neurons (Supplementary Fig. 2a,b, and see anatomical description of the HPs in ref. 4).

Act $\beta$ signalling promotes blood cell proliferation and adhesion. Next we investigated the effects of Act $\beta / \mathrm{dSmad} 2$ pathway lossand gain of function (lof, gof). Studying the viable null mutant $A c t \beta^{E D 80}$ (ref. 21), or silencing Act $\beta$ by in vivo RNAi, driving transgene expression ubiquitously or in sensory md neurons, we found diminished hemocyte numbers in the segmental HPs (Fig. 2a-e). Consistent with this, we observed an increase in the fraction of circulating hemocytes (Fig. 2f), and overall reduced total hemocyte numbers per larva (Fig. 2g), using a method of quantitative hemocyte retrieval from single Drosophila larvae ${ }^{22}$. These defects resembled hemocyte phenotypes seen on PNS neuron ablation ${ }^{4}$ and suggested defects in hemocyte adhesion, proliferation and/or survival. Silencing of $A$ ct $\beta$ in motor neurons using the driver OK6-GAL4 (Supplementary Fig. 2d,e,h,i) did not affect the localization of hemocytes in the HPs (Supplementary Fig. 2f,g,j,k), and resulted only in minor reductions of total hemocytes during various time points of larval development, which were non-significant according to Student's $t$-test (Supplementary Fig. 21). Act $\beta^{E D 80}$ mutants showed partial penetrance (62\% mutant phenotype) and were analysed sideby-side with controls as 2 nd instar larvae, to avoid compensatory mechanisms that became evident in 3rd instar larvae. In all backgrounds of $A c t \beta$ lof, PNS neuron clusters were present and appeared normal by cell number and dendritic morphology. This suggested a role for Act $\beta$ signalling in hemocytes, rather than indirect effects due to roles of Act $\beta$ in the nervous system $^{11,15,18,19}$.

To substantiate the role of $A c t \beta / \mathrm{dSmad} 2$ signalling in hemocytes, we systematically determined the effects of hemocyte-autonomous RNAi silencing of the Activin type II receptor punt (put) $)^{23}$ and the signal transducer $d S \operatorname{mad} 2$ (ref. 11). Similar to the loss of Act $\beta$ in the microenvironment, knockdown of Act $\beta$ pathway components in hemocytes resulted in diminished numbers of resident hemocytes in the segmental HPs (Fig. 2h-k). Silencing of the Activin type I receptor baboon $(b a b o)^{11}$ showed similar albeit milder effects on hemocyte localization. Silencing of the pathway in hemocytes by knockdown of put or dSmad2 further resulted in increased fractions of circulating hemocytes (Fig. 2l) and reduced total hemocyte numbers per larva (Fig. $2 \mathrm{~m}$ ). With prolonged RNAi silencing of put and $d S m a d 2$, the reduction in total hemocytes eventually reversed in older larvae (Supplementary Fig. 3), again implying putative compensatory mechanisms that take effect in the prolonged absence of dSmad2 signalling. Silencing of put and dSmad2 under the control of Hml - -GAL4 had no effect on lymph gland hemocytes in larvae of the developmental window studied (Supplementary Fig. 4a-i), and no concomitant increase in the fraction of crystal cells, neither of the lymph gland nor the embryonic lineage of hemocytes, was observed (for lymph gland see Supplementary Fig. 4c,f,i). This was examined because in the larva, plasmatocytes are known to give rise to small numbers of crystal cells ${ }^{24,25}$. Taken together, our findings suggest that sensory neuron-produced Act $\beta$ signals through the dSmad2 pathway in hemocytes, which supports hemocyte numbers and promotes hemocyte localization to the HPs.

To determine whether Act $\beta / \mathrm{dSmad} 2$ signalling has trophic and/or proliferative roles in hemocytes, we focused on the effects of $A c t \beta$ overexpression and babo gain of function. Moderate $A c t \beta$ overexpression in PNS neurons, or ectopic sites in oenocytes and epidermis using the driver Spalt (Sal)-GAL4, resulted in increased total hemocyte numbers per larva (Fig. 3a-g), while silencing of $A c t \beta$ in these locations had no significant effect according to
Student's $t$-test (Supplementary Fig. $5 \mathrm{a}-\mathrm{c}$ ). The increase in total hemocyte numbers on Act $\beta$ overexpression was accompanied by increased in vivo EdU incorporation, suggesting enhanced hemocyte proliferation (Fig. 3i). Consistently, silencing of put and $d S m a d 2$ in hemocytes resulted in reduced EdU incorporation of hemocytes in vivo (Fig. 3j). To substantiate a direct role of Act $\beta$ in hemocyte proliferation, we also examined larval hemocytes ex vivo under conditions of Act $\beta$ stimulation. Indeed, we found that Act $\beta$ promoted EdU incorporation, indicative of increased hemocyte proliferation (Fig. 3k). In contrast, high overactivation of the pathway by hemocyte specific expression of the constitutively activated receptor $b a b o-C A$ resulted in reduced hemocyte numbers per larva (Supplementary Fig. 6a-c) and drove hemocytes into apopotosis (Supplementary Fig. 6d). Neither Act $\beta$ overexpression nor dSmad2 pathway silencing in hemocytes increased the rate of hemocyte apoptosis (Supplementary Fig. $6 c, d$ ). Based on this, we conclude that the level of Act $\beta / \mathrm{dS}$ mad 2 signalling may determine the nature of the hemocyte response. At moderate activation levels, Act $\beta / \mathrm{dSmad} 2$ signalling is a positive regulator of hemocyte number that promotes proliferation, while high overactivation of the pathway drives cells into apoptosis. Consequently, we anticipate that the amplitude of $A c t \beta$ expression is likely to be tightly regulated.

Next we examined the role of Act $\beta / \mathrm{dSmad} 2$ signalling in hemocyte localization. Ectopic expression of Act $\beta$ in areas typically devoid of hemocytes, such as the Sal expressing ventral areas of the epidermis and oenocytes (Fig. 3e,f) or imaginal discs, did not result in a uniform adhesion or attraction of hemocytes, i.e., in the alternating gap areas of the epidermis where no sensory neuron clusters are located (Fig. 3e,f). Further, uniform overactivation of the pathway by hemocyte expression of babo-CA showed a largely unaffected overall pattern of resident hemocytes, despite the above-mentioned apoptosis of hemocytes (Supplementary Fig. 6a,b). This argued against a function of Act $\beta$ in hemocyte chemoattraction by gradient formation and led us instead to focus on a potential role of Act $\beta$ in the induction of hemocyte adhesion. Ectopic expression of $A c t \beta$ produced an overall trend of decreasing the fraction of circulating hemocytes, although this effect remained statistically insignificant by $t$-test (Fig. $3 \mathrm{~h}$ ), and accumulation of hemocytes in ectopic areas seemed minimal (Fig. 3c-f). Seeking a more sensitive assay to quantify hemocyte adhesion, we took advantage of the fact that resident hemocytes of the HPs can be mobilized by mechanical disturbance and spontaneously re-adhere to the body wall within $30-45 \mathrm{~min}$ (ref. 4). Using an established protocol for this assay ${ }^{22}$, we examined the adhesive properties of hemocytes under various Act $\beta / \mathrm{dSmad} 2$ pathway conditions. Indeed, dSmad2 pathway knockdowns in hemocytes, or Act $\beta$ silencing in PNS neurons, diminished hemocyte re-adhesion as evidenced by increased fractions of circulating hemocytes (Fig. 4a). However, dSmad2 pathway activation did not promote increased re-adhesion, suggesting an additionally needed, rate-limiting step in hemocyte adhesion. Moreover, we found that hemocyte-autonomous knockdowns of dSmad2 pathway components, but not Act $\beta$ silencing in neurons, produced a lack in hemocyte cluster formation and self-adhesion (Fig. 4b-e). Taken together, we conclude that the Act $\beta / \mathrm{dSmad} 2$ pathway directly or indirectly promotes hemocyte adhesion to the microenvironment (Fig. 4f, Model), a process that we predict requires in addition another, rate-limiting step. dSmad2 signalling may have an additional autonomous roles in hemocyte clustering/selfadhesion, which is revealed only when the pathway is blocked in hemocytes, thereby excluding alternative signalling by other Act family ligands as might be the case in Act $\beta$ lof backgrounds. 


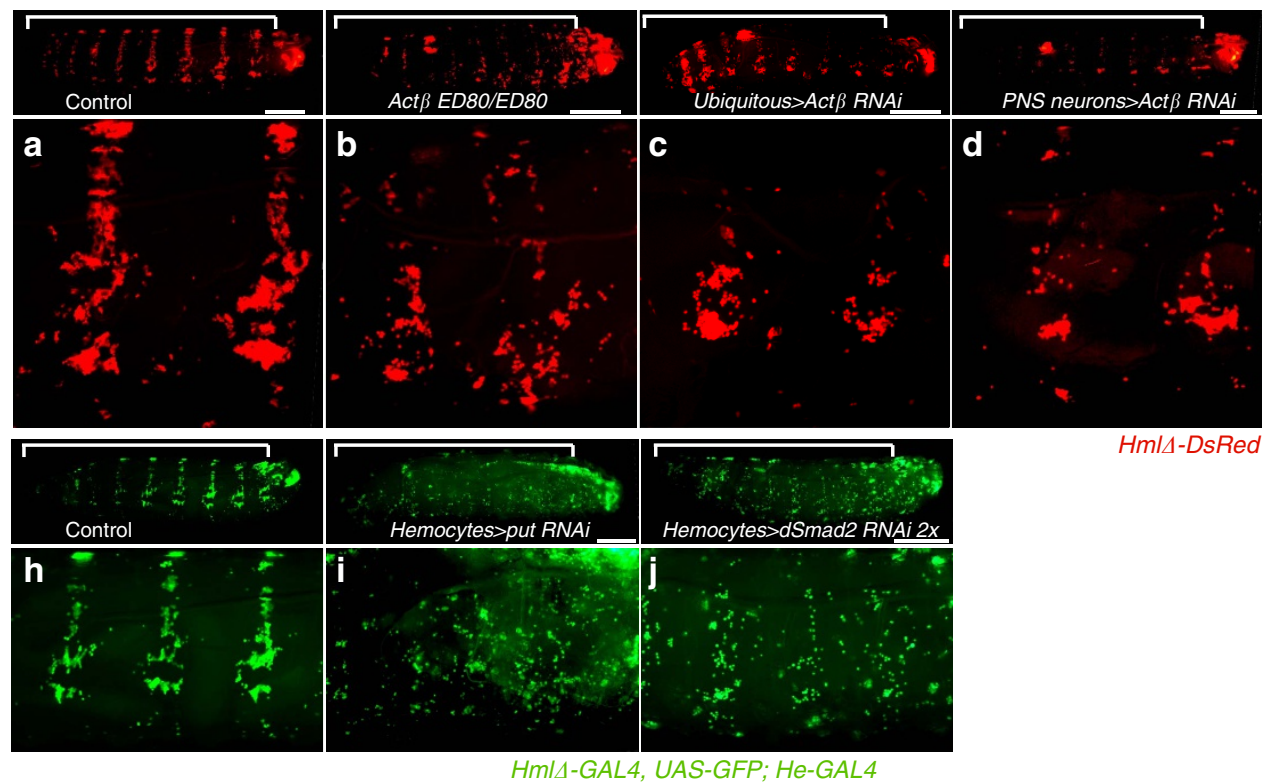

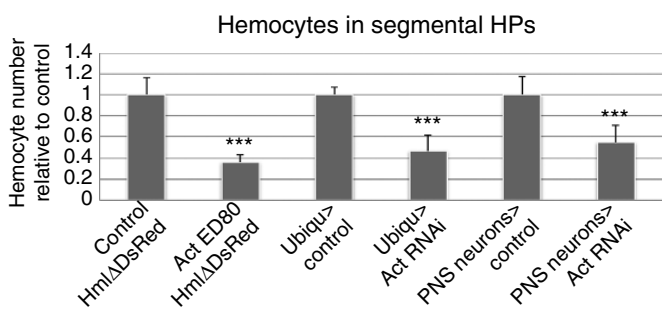

f

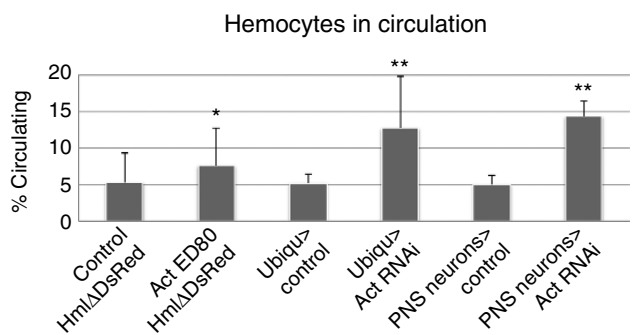

g

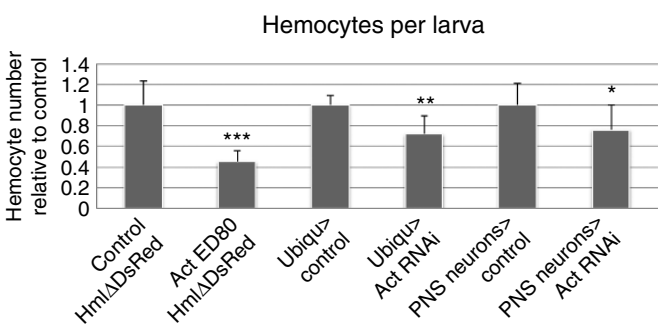

$\mathbf{k}$

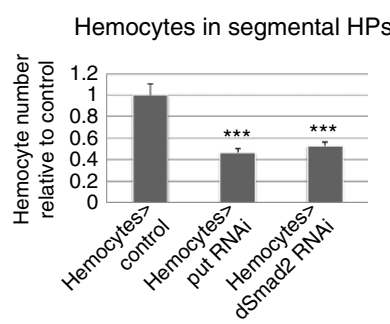

I

Hemocytes in circulation

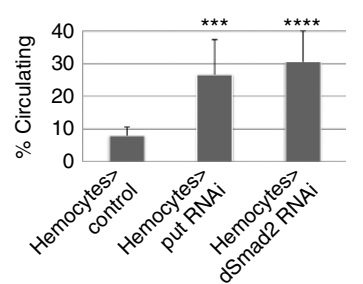

$\mathbf{m}$

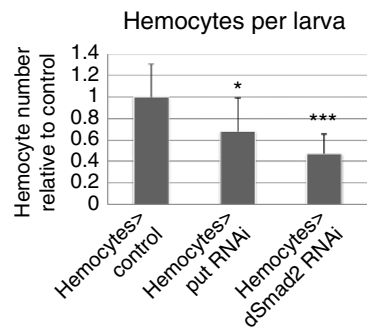

Figure 2 | Loss of Activin- $\boldsymbol{\beta} / \mathbf{d S m a d} \mathbf{2}$ signalling results in hemocyte defects. (a-d) Loss of Act $\beta$ in neurons affects hemocytes, lateral view of larvae (top panels) with close-up (lower panels). (a) Control Hml $\Delta$-DsRed / + (b) Act $\beta$ mutant, Hml $\Delta$-DsRed/ $+;$ Act $\beta$ ED80/ED80. (c) Act $\beta$ RNAi silencing with ubiquitous driver Actin5C-GAL4. (d) Act $\beta$ RNAi (UAS-Act RNAi Vienna GD) silencing in PNS neurons, md neuron specific driver 21-7-GAL4. (e) Hemocyte counts in segmental HPs (bracketed areas in a-d top panels), $n=4-7$ per genotype. (f) Fraction of circulating hemocytes, $n=4$ to 8 per genotype. (g) Total hemocyte counts per larva, $n=4$ to 8 per genotype. In e-g experiment numbers relative to matching control cohorts, genotypes Hml 4 -DsRed/ $+;$ Act $\beta$ ED80/ED80 (matching control:

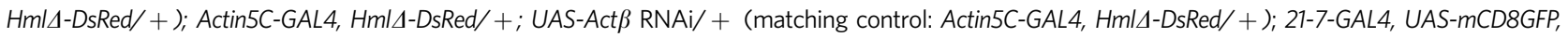

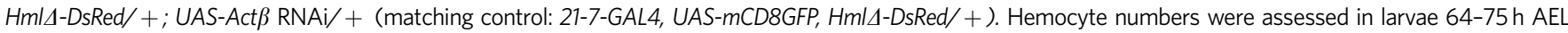
(2.2-2.6 mm) (h-j) Hemocyte-specific RNAi silencing of Act $\beta$ pathway components, driver HmlA-GAL4, UAS-GFP; He-GAL4. Top panels whole larva lateral view, lower panels closeups. (h) Control; (i) UAS-put RNAi; (j) UAS-dSmad2 RNAi. (k) Hemocyte counts in segmental HPs (bracketed areas h-j in top panels), $n=4$ to 7 per genotype. (I) Fraction of circulating hemocytes, $n=7$ to 10 per genotype. (m) Total hemocyte counts per larva, $n=7$ to 11 per genotype; larvae 53-60 h AEL (1.8-2.0 mm). In (k-m) experiment numbers relative to matching control cohorts are shown; genotypes Hml 4 -GAL4, UAS-GFP/ +; He-GAL4/ + (control); HmlAGAL4, UAS-GFP/ + ; He-GAL4/UAS-put RNAi or HmIA-GAL4, UAS-GFP/UAS-dSmad2 RNAi; He-GAL4/ + (experiment). Scale bars:, a-d and i,j 0.5 mm. Error bars represent s.d., and two-tailed $t$-test values correspond to NS (not significant) $P>0.05 ;{ }^{\star} P \leq 0.05 ;{ }^{\star \star} P \leq 0.01 ;{ }^{\star \star \star} P \leq 0.001 ;{ }^{\star \star \star \star} P \leq 0.0001$. 

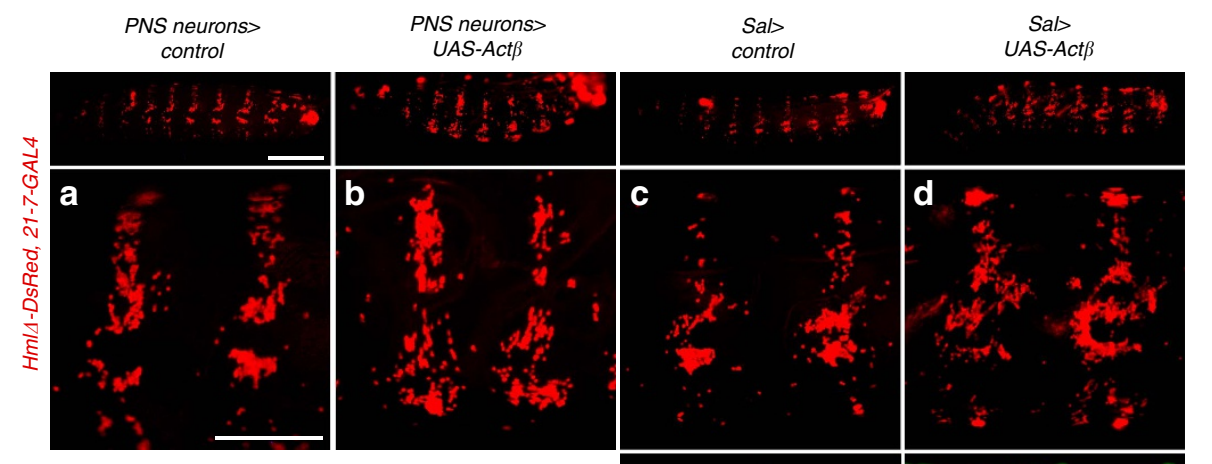

i

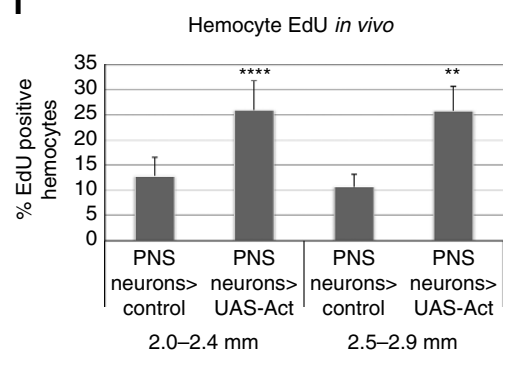

g

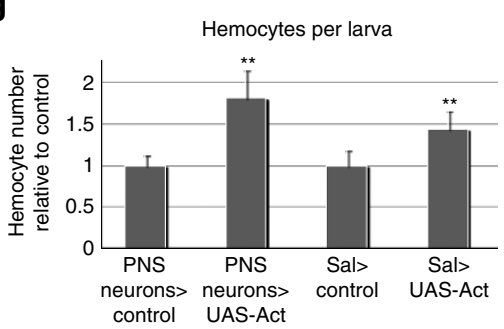

h
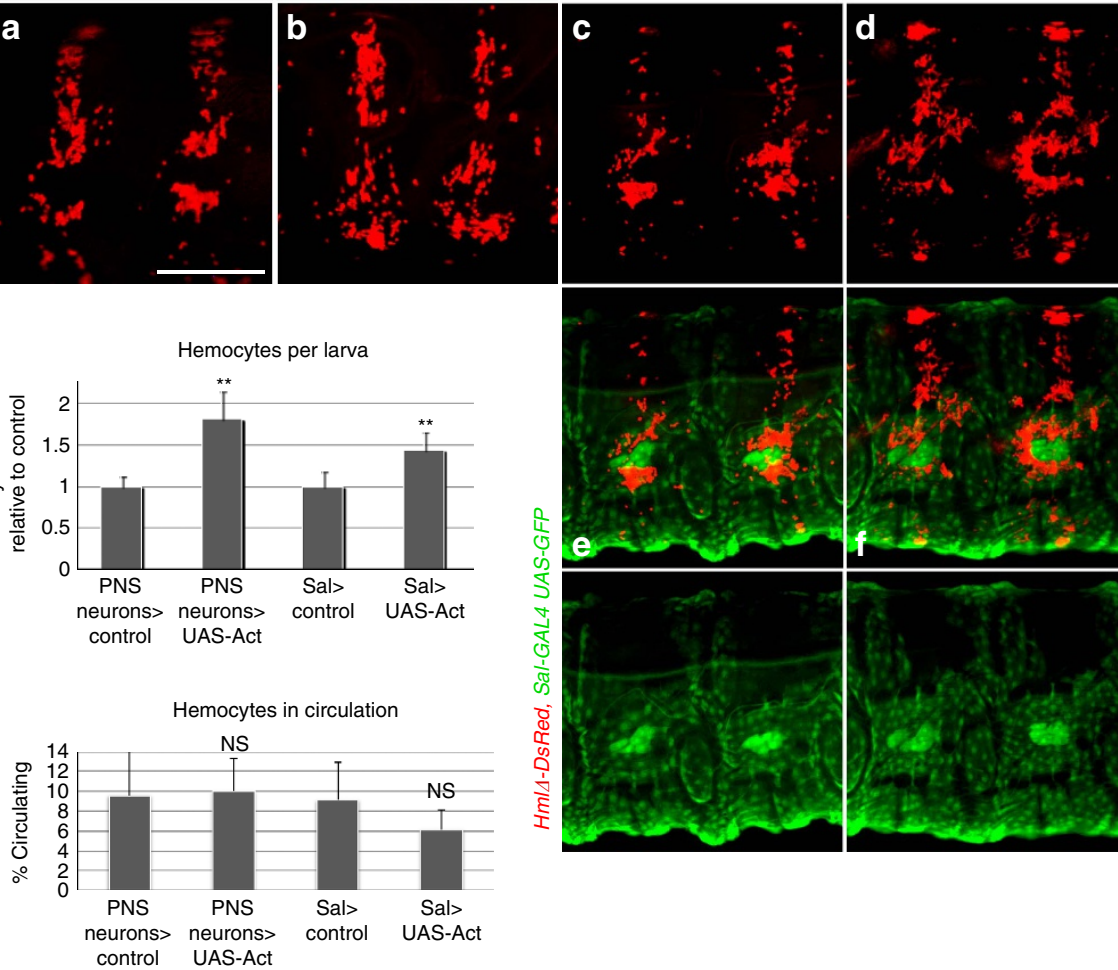

j

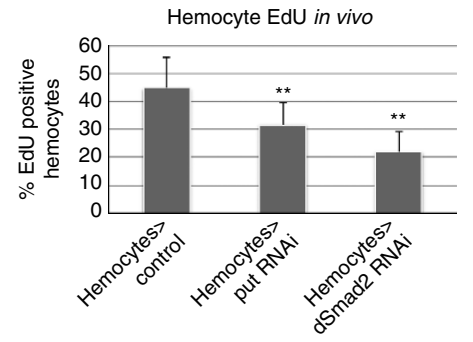

k

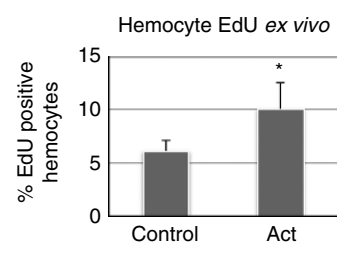

Figure 3 | Activin- $\beta$ promotes hemocyte proliferation. (a,b) Control (Hml $-D s R e d(r e d)$, and overexpression of Act $\beta$ in PNS neurons by 21-7-GAL4 with hemocytes Hml $\Delta$-DsRed (red). Whole larvae lateral view (top panel), and closeup (lower panel). (c,d) Control and ectopic expression of Act $\beta$ in ventral epidermis and oenocytes using the driver Sal-GAL4 (GMR85E05-GAL4) marked by UAS-GFP (green), and hemocytes HmlA-DsRed (red). Whole larvae lateral view (top panel), and closeup (lower panel). (e,f) Same as in (c,d) but combined (red, green) and single channels (green) of close-up images shown. (g) Total hemocyte counts per larva, genotypes are 21-7-GAL4, UAS-mCD8GFP, HmlA-DsRed/ +; UAS-Act $\beta$ / + (control is 21-7-GAL4, UAS-mCD8GFP,


GAL4, UAS-GFP/ +),$n=5$ to 6. (h) Hemocytes in circulation, genotypes and $\mathrm{n}$ as in $\mathbf{g}$. (i) In vivo EdU incorporation, genotypes 21-7-GAL4, UAS-mCD8GFP, $\mathrm{Hml}$-DsRed/ + (control) and 21-7-GAL4, UAS-mCD8GFP, HmlA-DsRed/ +; UAS-Act $\beta$ (III) $/+; n=36$ for larvae 60-70 h AEL $(2.0-2.4 \mathrm{~mm})$, and $n=8 \mathrm{for}$ larvae 71-83 h AEL (2.5-2.9 mm). (j) In vivo EdU incorporation, Hml - -GAL4, UAS-GFP; He-GAL4 x yw (control); x UAS-put RNAi and UAS-dSmad2 RNAi (experiment larvae); $n=6$; larvae 46-52 h AEL (1.5-1.7 mm). (k) EdU incorporation of released larval hemocytes stimulated with Act $\beta$ ex vivo; genotype Hml 4 GAL4, UAS-GFP; He-GAL4; average over 4 independent experiments (Act $\beta+/-$ conditioned media from 2 cell lines in 2 replicates each). Scale bars, a (upper panel), $0.5 \mathrm{~mm}$; a (lower panel), $0.2 \mathrm{~mm}$. Error bars represent s.d., and two-tailed $t$-test values correspond to NS (not significant) $P>0.05$; ${ }^{\star} P \leq 0.05$; ${ }^{\star \star} P \leq 0.01 ;{ }^{\star \star \star} P \leq 0.001 ;{ }^{\star \star \star \star} P \leq 0.0001$.

Act $\beta$ links sensory neuron activity with blood cell responses. Considering the anatomical contacts of PNS neurons with hemocytes, and the role of Act $\beta$ as neuron-emanating signal that supports hemocyte proliferation and adhesion, we sought to determine whether PNS neuron activity may regulate blood cell behaviours. Using a diagnostic driver for acetylcholine production, Cha-GAL4, we confirmed previous reports that PNS neurons are cholinergic (Fig. 5a,b) ${ }^{26}$. This allowed us to use the pan Acetylcholine Receptor (AChR) agonist carbachol (carbamoylcholine) for the stimulation of larval sensory neurons. Interestingly, we found that cuticle exposure of intact larvae to carbachol promoted short-term recruitment of hemocytes to the HPs within $15 \mathrm{~min}$, resulting in an enhanced resident hemocyte pattern (Fig. $5 c, d$ ). Since carbachol may not target neurons of the PNS only, we confirmed our findings by genetic manipulation of the PNS. Using the PNS neuron-specific driver 21-7-GAL4 that is nearly devoid of CNS expression, we transiently silenced sensory neurons using two methods; (1) hyperpolarization through transient expression of the inward rectifying $\mathrm{K}^{+}$channel Kir2.1 (ref. 27), or (2) transient interference with neuronal endocytosis and synaptic vesicle recycling through expression of the temperature-sensitive dominant-negative dynamin UAS-shi $d n^{\text {ts }}$ (ref. 28). Indeed, both sensory neuron manipulations strongly and reversibly disturbed the pattern of larval resident hemocytes within a short-term $2 \mathrm{~h}$ time frame, indicative of compromised hemocyte adhesion to the HPs (Fig. 5e-g). As a control, we expressed the same transgenes in glia using repo-GAL4 (ref. 29), yet no hemocyte phenotypes were observed (Supplementary Fig. 7a-d), demonstrating specificity of the observed hemocyte responses to neuronal silencing.

In addition to these short-term ( $15 \mathrm{~min}-2 \mathrm{~h}$ ) effects on hemocyte localization to the HPs, we examined the long-term effects of stimulation by carbachol, or neuronal silencing by Kir2.1 or UASshi $d n^{t s}$, on hemocytes. Interestingly, we found that carbachol exposure over several days of larval development significantly increased total hemocyte numbers per larva compared to controls according to Student's $t$-test (Fig. 5h). Consistently, silencing of PNS neurons through various regimens of heat shock induction of Kir2.1 or the dominant-negative dynamin shi $d n^{t s}$ over $20-48 \mathrm{~h}$ 
a

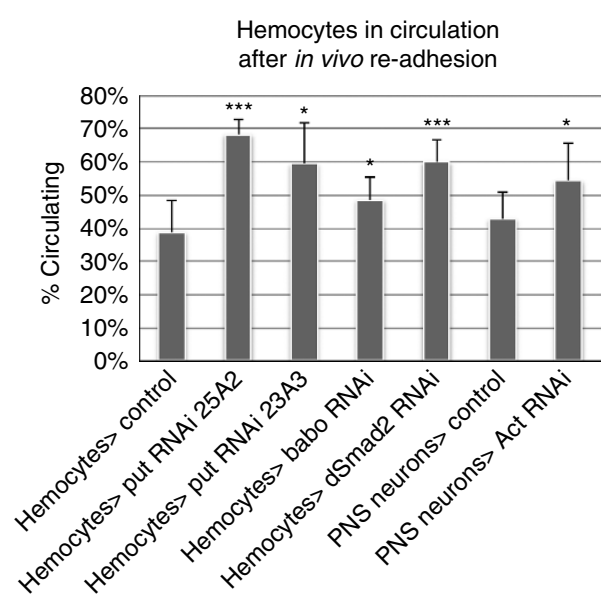

f

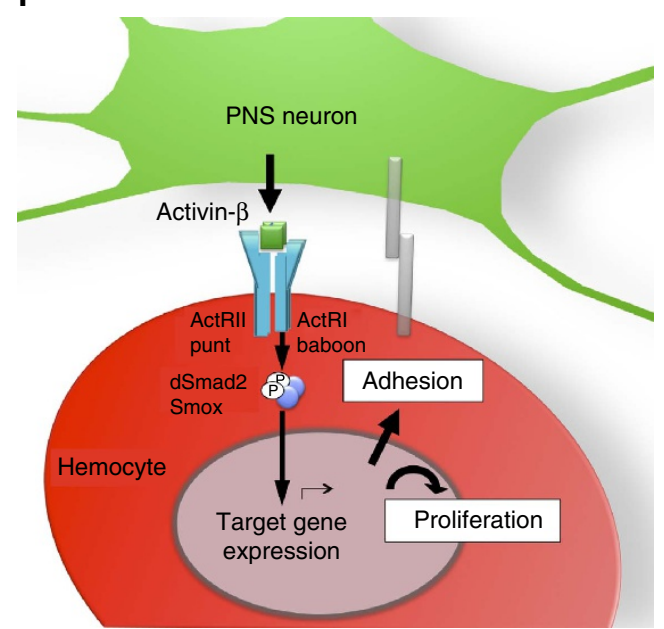


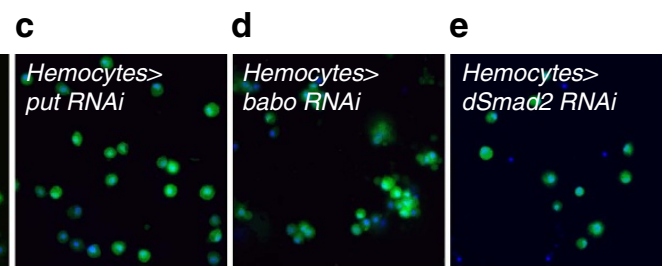

HmIS-GAL4, UAS-GFP; He-GAL4 DAPI

Figure 4 | Activin- $\boldsymbol{\beta}$ promotes hemocyte adhesion. (a) In vivo re-adhesion of hemocytes after mechanical disturbance, bars illustrate percent of circulating hemocytes; genotypes are HmlA-GAL4, UAS-GFP; He-GAL4 × UAS-RNAi transgenes and control; and 21-7-GAL4, UAS-mCD8GFP, HmlA-DsRed $\times$ UASAct $\beta$ RNAi and control; $n=7$ to 8. (b-e) Ex vivo released hemocytes show different levels of aggregation; HmlA-GAL4, UAS-GFP; He-GAL4 $\times$ (b) Control; (c) UAS-put RNAi; (d) UAS-babo RNAi; (e) UAS-dSmad2 RNAi. (f) Model of Act $\beta$ production by sensory neurons and induction of hemocyte responses in proliferation and adhesion. Scale bars, b, $50 \mu \mathrm{m}$. Error bars represent s.d., and two-tailed $t$-test values correspond to NS (not significant) $P>0.05$; ${ }^{\star} P \leq 0.05 ;{ }^{\star \star} P \leq 0.01 ;{ }^{\star \star \star} P \leq 0.001 ;{ }^{\star \star \star \star} P \leq 0.0001$.

had opposite effects, resulting in larvae with reduced total hemocyte numbers (Fig. 5h). To examine whether the carbacholinduced increase in hemocyte number was based on proliferation, we quantified in vivo EdU incorporation of hemocytes. Indeed, carbachol-exposed larvae showed about 1.5-fold increased EdU incorporation over controls, an effect which interestingly was mainly seen in younger 2nd instar larvae (Fig. 5i). Further, carbachol-exposed larvae showed an overall larger increase in the resident hemocyte population of the hematopoietic sites compared to the circulating fraction (Fig. 5j), consistent with our model proposing enhanced proliferation of resident hemocytes in the HPs. Taken together, our data suggest that PNS neuronal activity supports hemocyte localization and expansion by proliferation, and predict a molecular signal from PNS neurons that is inducible by sensory activity.

To determine whether Act $\beta$ might play a role as this inducible signal, we quantified Act $\beta$ expression in sensory neurons of varying states of activity. Indeed, exposure of intact Drosophila larvae to carbachol induced a substantial and dose-dependent increase in $A c t \beta$ expression within $2-3 \mathrm{~h}$, as quantified by the expression of a UAS-luciferase transgene driven by the Act $\beta$ GAL4 reporter (Fig. 6a). For this experiment, we focused on reporter expression in the PNS by carefully removing the CNS before luciferase quantification. To examine whether the increase in Act $\beta$-GAL4 expression was specific to sensory neurons, we coexpressed a UAS-GFP transgene. GFP imaging of freshly dissected larvae confirmed increased expression in PNS neurons, and did not show signs of ectopic expression in unrelated tissues (Fig. 6b-d). In a converse experiment, we examined whether inducible expression of Kir2.1 affects expression of UAS-GFP by the reporter Act $\beta$-GAL4 in PNS neurons. Inducing expression (through $t u b-G A L 80^{t s}$ ) in limited time windows of $22 \mathrm{~h}$ to visualize GFP expression yet avoiding lethality by high expression of Kir2.1, we observed induction of GFP in Act $\beta$ expressing PNS neurons of controls, while this GFP signal was largely absent in parallel cohorts silenced by coexpressed Kir2.1 (Fig. 6e-g). In addition to its effects on the PNS, Kir2.1 coexpression also reduced $A c t \beta$-GAL4 driven GFP expression in the CNS (Fig. 6f,g).

Finally, we asked whether Act $\beta$ is required for the induction of hemocyte responses on stimulation with carbachol. Comparing $A c t \beta^{E D 80}$ null mutant larvae with $\operatorname{Act} \beta$-competent controls, we found that loss of $A c t \beta$ attenuated the effect of carbachol-induced blood cell expansion (Fig. 6h). This suggested that Act $\beta$ plays a major role in the cholinergic regulation of hemocytes, consistent with a model of neuronal activity-induced $\operatorname{Act} \beta$ expression (Fig. 6k). However, Act $\beta$ mutants showed mild albeit by $t$-test insignificant carbachol-induced blood cell expansion (Fig. 6h) and partial short-term hemocyte recruitment to the HPs on $15 \mathrm{~min}$ of carbachol exposure (Fig. 6i,j), suggesting additional inducible signal/s that may contribute to these effects. Taken together, our findings support a model in which PNS neuronal activity promotes Act $\beta$ expression, which in turn drives hemocyte expansion and long-term localization to the HPs (Fig. 6k).

\section{Discussion}

This research identified $A c t \beta$ as one of the elusive genes that govern hemocyte proliferation in the hematopoietic sites (HPs) of the Drosophila larva, as was predicted by previous functional studies ${ }^{4}$. Our work further links Act $\beta$ RNA expression to the level of PNS neuronal activity. This model implies that increased expression of $A c t \beta$ would give rise to higher levels of active $\operatorname{Act} \beta$ 

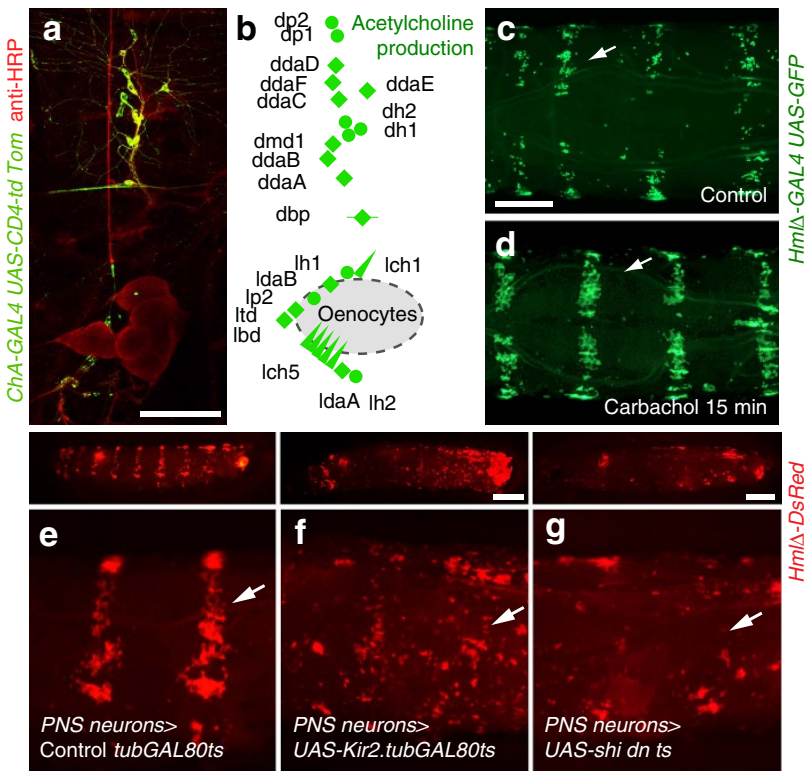

h

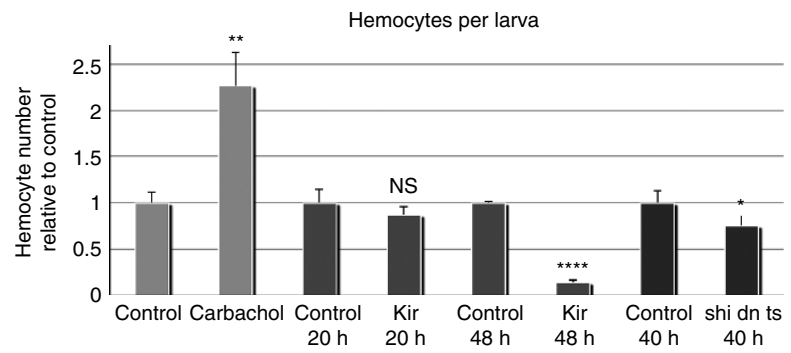

i

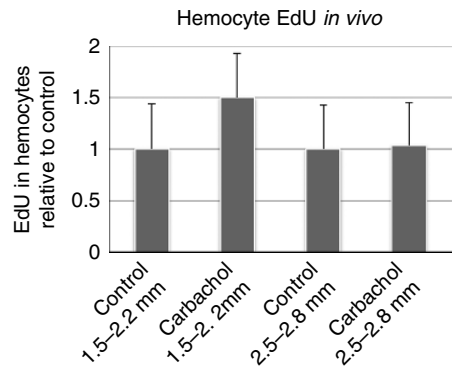

j

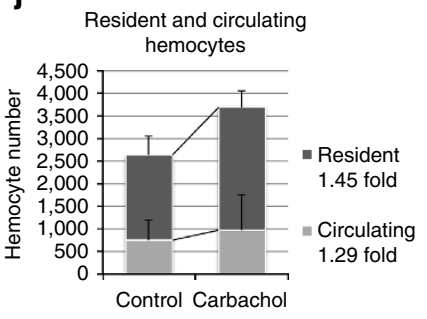

Figure 5 | PNS neuron activation promotes hemocyte recruitment and proliferation. (a) Labelling of cholinergic PNS neurons using the diagnostic driver Cha-GAL4 x UAS-CD4-td Tom and anti-HRP 488; false colouring of ChA positive neurons in green, HRP in red. (b) Model, Cha-GAL4 positive signal in all PNS neurons. (c,d) Stimulation of PNS neurons. Live imaging of larvae marked with HmlA-GAL4, UAS-EGFP, dorsal view. (c) Control; (d) carbachol, shortterm exposure $\left(10 \mathrm{mg} \mathrm{ml}^{-1}, 15 \mathrm{~min}\right)$. (e-g) Live imaging of hemocytes (Hm/ $-\mathrm{DsRed}$, red) after transient disturbance of electrochemical signalling in PNS neurons, hemocytes in red; arrows point to HPs. Larvae lateral view (top panels), closeups (lower panels). (e) Control, 21-7 GAL4, UAS-mCD8GFP, Hml DsRed/ + ; (f) Kir2.1 expression to hyperpolarize neurons, 21-7 GAL4, UAS-mCD8GFP, HmlA-DsRed/ tubGAL80ts; UAS-Kir2.1/+; (g) Expression of UAS-shi $d n^{t s}$ in neurons, 21-7 GAL4, UAS-mCD8GFP, HmlA-DsRed/ +; UAS-shi $\mathrm{dn}^{\text {ts }} /+$. (h) Larvae $-/+$ carbachol exposed from hatching onward, and larvae $-/+$ genetic neuronal silencing as in $(\mathbf{f})$ and $(\mathbf{g})$, heats shocks to induce transgenes were applied as indicated; total hemocyte counts from single larvae (2nd instars, 66-78 h AEL corresponding to 2.3-2.7 mm). For each experimental cohort, total hemocyte numbers of experiment sets relative to side-by-side control sets of larvae are shown; $n=3$ to 5 and comparable effect in independent repeats. (i) In vivo EdU incorporation of hemocytes from larvae exposed to $-/+$ carbachol $\left(0.7 \mathrm{mg} \mathrm{ml}^{-1}\right.$ ) from 1st instar ( $22 \mathrm{~h} \mathrm{AEL)} \mathrm{onward.} \mathrm{Genotype} \mathrm{is} \mathrm{Hml}-$-GAL4, UAS-GFP; He-GAL4; larval sizes as indicated, corresponding to 2nd instar 47-65 h AEL (1.5-2.2 mm) and 72-80 h AEL (2.5-2.8 mm). EdU incorporation of experiment sets relative to side-by-side control sets of larvae are shown; average of three independent experiments, total $n=6$ to 9 per condition. (j) Fraction of resident and circulating hemocytes of $-/+$ carbachol treated larvae $74-85 \mathrm{~h} \mathrm{AEL}(2.6-3.0 \mathrm{~mm})$, on carbachol $\left(0.7 \mathrm{mg} \mathrm{ml}^{-1}\right.$ ) since first instar ( 22 h AEL); genotype Hml 4 -GAL4, UAS-GFP; He-GAL4. Carbachol treated animals show a larger increase in the resident than in the circulating population ( 1.45 fold versus 1.29 fold); $n=13$ to 14 per condition. Scale bars, $\mathbf{a}, 100 \mu \mathrm{m} ; \mathbf{c}, 0.2 \mathrm{~mm}$; and $\mathbf{f}, \mathbf{g}, 0.5 \mathrm{~mm}$. Error bars represent s.d., and two-tailed $t$-tests value correspond to NS (not significant) $P>0.05 ;{ }^{\star} P \leq 0.05 ;{ }^{\star \star} P \leq 0.01 ;{ }^{\star \star \star} P \leq 0.001 ;{ }^{\star \star \star \star} P \leq 0.0001$.

protein, although the formal demonstration awaits development of a suitable tool for the detection of Act $\beta$ protein. In the future, it will be interesting to study specific sensory stimuli that trigger hemocyte responses. Sensory neurons of the PNS have a prime function in detecting innocuous and noxious sensory stimuli such as mechanical strain, temperature, chemicals and light ${ }^{30,31}$, many of which signal potentially harmful conditions that may cause tissue damage. Thus, linking the detection of challenging conditions with the adaptive expansion of the blood cell pool may be an efficient system to elevate the levels of macrophages, to remove and repair damaged tissues, enhancing the overall fitness of the animal. Because Drosophila larval hemocytes persist into the adult stage ${ }^{6,7}$, the mechanism of sensory neuron-induced blood cell responses may allow adaptation of the animal beyond the larval stage.

In Drosophila self-renewing hemocytes, Act $\beta / \mathrm{dSmad} 2$ signalling has diverse effects on proliferation, apoptosis and adhesion. Our ex vivo data indicate that hemocyte proliferation is likely a direct effect, which is consistent with similar roles of babo/ $\mathrm{dSmad} 2$ in other tissues such as Drosophila imaginal discs and brain $^{11,21}$, and TGf- $\beta$ family dependent proliferation in vertebrate systems ${ }^{32,33}$. Echoing our findings of babo-CA driven hemocyte apoptosis, TGF- $\beta$ family mediated direct or indirect effects on apoptosis have been described in invertebrate and vertebrate systems $^{33,34}$. Overall, TGF- $\beta$ family signalling is known for its multifaceted biological roles, depending on the cellular contexts and levels of ligand stimulation, which often translate into qualitatively distinct transcriptional and other cellular responses, that are mediated by both Smad and non-Smad signalling mechanisms ${ }^{32,33}$. While Drosophila Act $\beta$ and possibly related TGF- $\beta$ family ligands are known to signal through the induction of ecdysone receptor $(E c R)$ in some but not all Drosophila tissues $^{15,35}$, we found no indication for a link with EcR expression in hemocytes, suggesting other signalling mechanisms in the regulation of larval blood cell responses. In the studied Drosophila system, it further remains to be seen whether $\operatorname{Act} \beta / \mathrm{dSmad} 2$ signalling has direct or indirect effects on hemocyte adhesion, and which other rate-limiting step/s may contribute to this process. Since hemocyte-autonomous loss of $\mathrm{d} S \operatorname{mad} 2$ signalling causes a more severe phenotype than Act $\beta$ lof, we speculate that other Act family ligands such as daw and myo, which are expressed in various tissues including surface glia, muscle, fat body, gut, and imaginal discs ${ }^{11,17,20,36,37}$ may partially substitute for $A$ ct $\beta$ in its absence. Overall, Act $\beta$ is likely to be only one player in a more complex regulatory network. Future research will identify other inducible signals from neurons that regulate neuron-blood cell communications. This is predicted from Act $\beta$ mutants that only partially block carbachol-induced blood cell responses. Act $\beta$ / $d S m a d 2$ lof and pathway silencing in hemocytes also reveal an underlying ability of the cells to compensate for the lack of this 
a

e

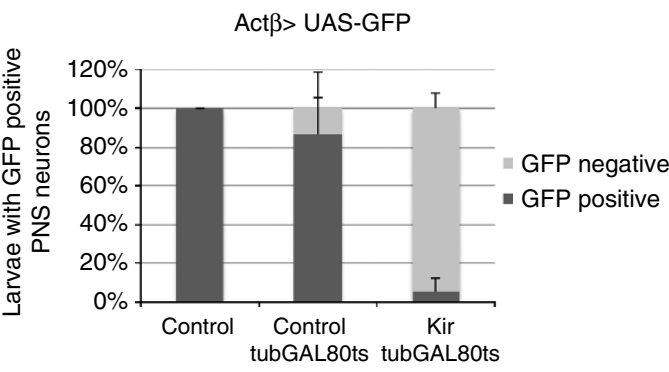

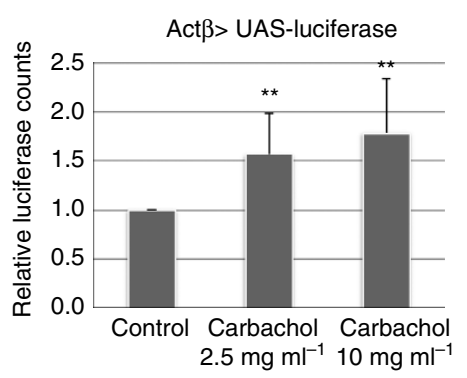
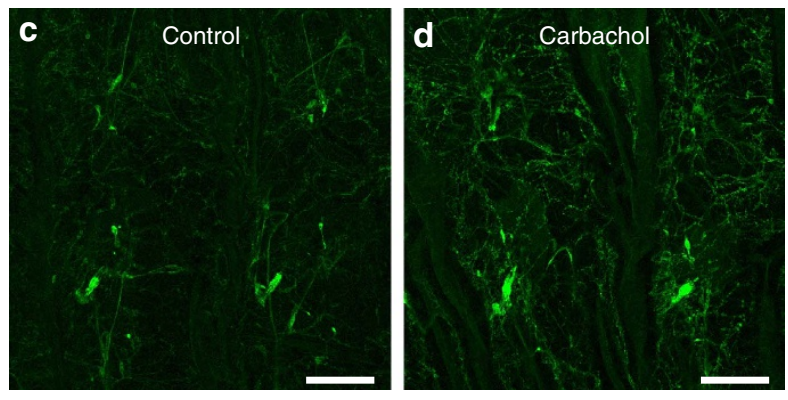

ActB-GAL4 UAS-GFP
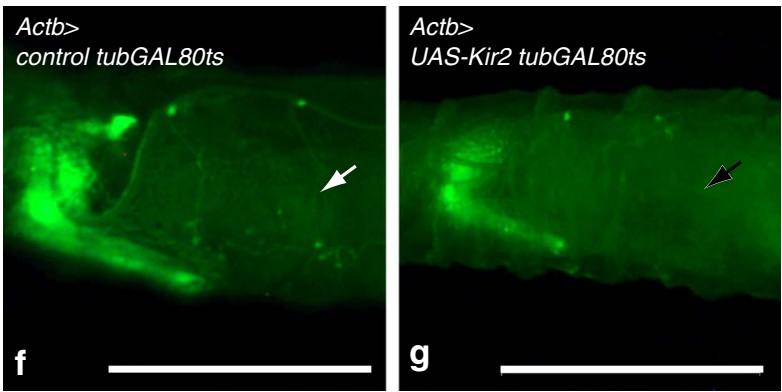

Actß-GAL4 UAS-GFP

h
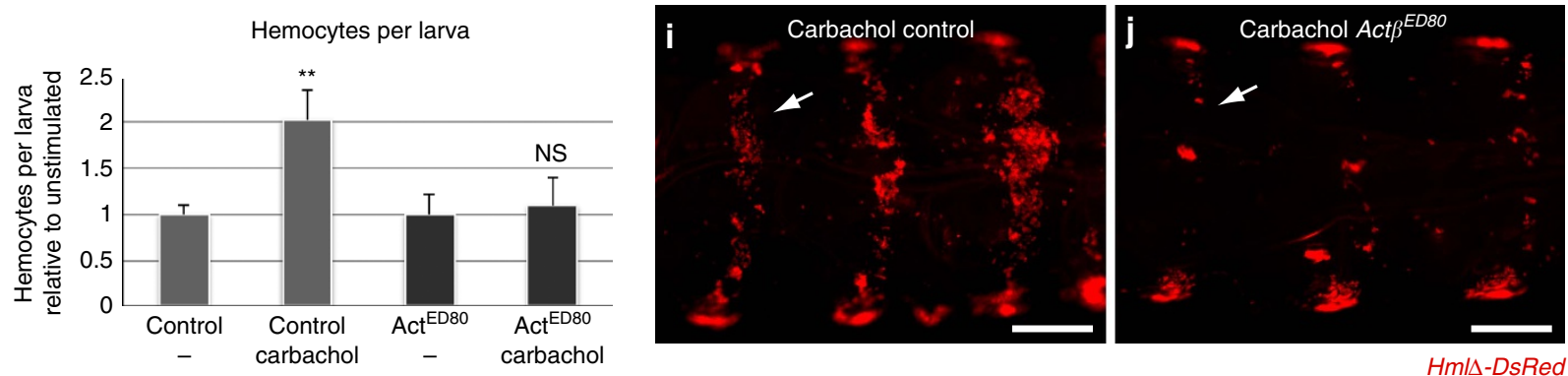

$\mathbf{k}$

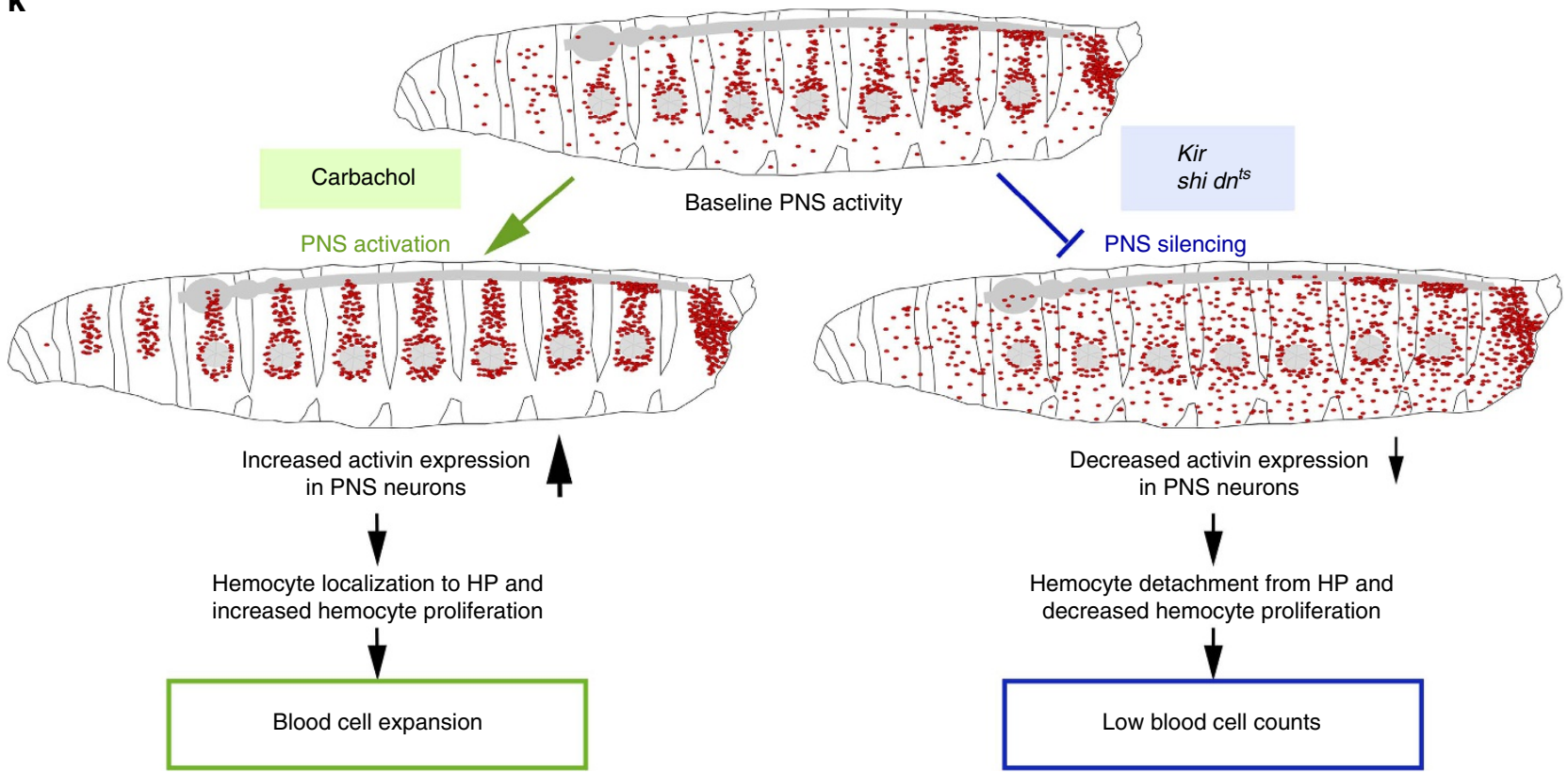

signalling pathway and the associated impairment in proliferation. Time course experiments with various RNAi lines suggest that the amplitude and temporal occurrence of the compensatory response may be proportional to the severity of the block in $\mathrm{d} S m a d 2$ signalling. Future investigation will address whether the related BMP/Mad pathway might play a part in this, as silencing of Mad in hemocytes appeared to dampen elevated hemocyte numbers seen in dSmad2 null mutants. Similar 
observations of $d S m a d 2$ lof causing Mad overactivation have been reported in the Drosophila wing disc and neuromuscular junction previously ${ }^{20,38}$.

Larval development may comprise distinct sensitive phases for the regulation of hemocyte responses. This is supported by carbachol promoting hemocyte proliferation preferentially in the early-mid 2nd instar larva, that is, at a stage when hemocytes are still tightly localized to the $\mathrm{HPS}^{4,22}$. Likewise, the effects of $A c t \beta$ lof and pathway silencing in hemocytes are more pronounced in younger larvae, suggesting a possible stronger dependence on the pathway, in addition to the emergence of compensatory mechanisms under lof conditions over time (above). Moreover, it will be interesting to investigate whether Act $\beta$ signalling may not only vary temporally, but also by the ability of cell types to produce active Act $\beta$ ligand, thereby influencing signalling outcomes, consistent with the cell type specific processing known for Activins and other ligands of the TGF- $\beta$ family in both invertebrates and vertebrates $16,39,40$.

Drosophila Act $\beta$ has previously been studied for its role in the formation and function of neuromuscular junctions in the Drosophila larva, where Act $\beta$ expressing motor neurons project axons from the CNS, reaching from the center of the larva to the muscle layers of the body wall ${ }^{16,17,20}$ However, resident hemocytes are shielded from these areas through the muscle layers of the body wall, which also form the base of the HPs, thereby creating an anatomical space between the muscle layers and epidermis ${ }^{4}$ where resident hemocytes and Act $\beta$ expressing sensory neurons colocalize (i.e., the Hematopoietic Pocket). The model that sensory neurons signal to adjacent hemocytes in the HPs is further supported by the fact that Act $\beta$ silencing in motor neurons did not affect resident hemocyte localization and had, by $t$-test, no significant effect on hemocyte numbers. However, we cannot completely rule out involvement of alternative or additional scenarios, for example, that experimental manipulations of PNS activity, which also feed back to the CNS, would in turn trigger a signal to motor neurons that may respond by secreting Act $\beta$ and/or another factor/s, thereby influencing hemocytes and/or the PNS itself. Likewise, although we confirmed the direct effect of Act $\beta$ on hemocytes ex vivo, and found no signs of altered sensory neuron morphology under $A c t \beta$ lof/silencing, we cannot rule out that in the larva, Act $\beta$ may contribute to molecular changes in the PNS that in turn might contribute to the observed hemocyte effects.

Sensory neurons of the HPs project axons to the $\mathrm{CNS}^{41}$, and our work shows that hemocytes are closely adjacent to and/or form direct contacts with sensory neurons, likely along the neuron cell bodies and dendrites, suggesting the communication involves non-canonical mechanisms. In Drosophila, as in vertebrates, signal transfer along all neuronal membrane surfaces, including dendritic synapses and dendrodendritic connections, have been described ${ }^{42,43}$, which may also form the interface in neuron-blood cell communication. The transcriptional induction of Act $\beta$ in response to sensory stimuli recalls previous reports of the transcriptional upregulation of Act $\beta$ in the formation of long-term memory in both flies and vertebrates ${ }^{44,45}$. This suggests parallels between the neuronal regulation within the CNS, and PNS-blood cell circuits, which will be an interesting subject for future study. Based on our findings and another recent report demonstrating that transcriptional regulation of the related BMP Decapentaplegic $(D p p)$ in the Drosophila wing epithelium depends on the $\mathrm{K}^{+}$ channel Irk2 (ref. 46), we propose that cellular electrochemical potential may be a more general theme in the expression of TGF- $\beta$ family ligands.

Our findings in the Drosophila model pioneer a new concept that has not been shown in any vertebrate system to date- the neuronal induction of self-renewing, tissue-resident blood cells. These cells correspond to the broadly distributed system of selfrenewing myeloid cells that are present in most vertebrate organs, which by lineage are completely independent from blood cell formation fueled by hematopoietic stem cells ${ }^{3-6,47,48}$. In vertebrates, TGF- $\beta$ family ligands such as Activin $A$ and TGF- $\beta$ regulate the activity and immune functions of macrophages, and cellular and humoral immune responses, in multiple ways through autocrine and paracrine signalling 49,50 . While the autonomic neuronal and glial regulation of hematopoietic stem and progenitor cells in the bone marrow has been recognized ${ }^{51-55}$, the role of sensory innervation in bone marrow hematopoiesis remains unknown. Even more so, nothing is known about the role of the nervous system in the regulation of the independent, self-renewing myeloid system of tissue macrophages. However, local neurons and sensory innervation of many organs including skin, lung, heart and pancreas ${ }^{56-59}$ and inducible changes in the self-renewal rates of tissue macrophages ${ }^{2}$, suggest that principles of neuronal regulation are likely also at work in vertebrates, providing a link between neuronal sensing and adaptive responses of local blood cell populations.

\section{Methods}

Fly strains. Drosophila lines used were HmlA-DsRed(2 copies)/CyO (ref. 4), $A c t \beta^{E D 80} /$ unc13GFP (ref. 21), tub-GAL80 ${ }^{\text {ts }}$ (ref. 60), Actin5c-GAL4 (Bloomington), Act $\beta$-GAL4 (ref. 18), daw-GAL4 (ref. 21), myo-GAL4 (ref. 13), Cha-GAL4 (Bloomington), en-GAL4 (Bloomington), Sal-GAL4 (GMR85E05-GAL4 insertion in

\footnotetext{
Figure 6 | Neuronal activity promotes Act $\boldsymbol{\beta}$ expression which drives hemocyte expansion. (a-d) Carbachol induces dose-dependent transcriptional induction of an Act $\beta$ reporter. (a) Quantification of luciferase expression in larval fillets of Act $\beta$-GAL4, UAS-CD4-tdGFP/UAS-luciferase animals, stimulated by increasing doses of carbachol as intact larvae $\left(2.5 \mathrm{mg} \mathrm{ml}^{-1} ; 10 \mathrm{mg} \mathrm{ml}^{-1}\right)$. Pools of $2-3$ larval fillets per sample in biological replicates; carbacholstimulated conditions were normalized to unstimulated condition (total $n=4-6$ per condition); statistics show average of four such normalized independent experiments. (b) Carbachol-induced increase of GFP expression in fillets of Act $\beta$-GAL4, UAS-CD4-tdGFP larvae, signal quantification over three independent sets of images, normalized to unstimulated condition and averaged $(n=3)$. (c,d) Sample set of images as in $\mathbf{b}$. (c) Control, (d) $4 \mathrm{~h}$ stimulation with carbachol as intact larva, $2.5 \mathrm{mg} \mathrm{ml}^{-1}$. (e-g) Transient PNS silencing with UAS-Kir2.1 reduces GFP expression; induction for $22 \mathrm{~h}$ at $27^{\circ} \mathrm{C}$. (e) Fraction of larvae showing visible GFP expression in PNS neurons, genotype tubGAL80 ts $/+$; Act $\beta$-GAL4, UAS-CD4-tdGFP/ + (control) and tubGAL80 ${ }^{\text {ts }} /+$; Act $\beta$-GAL4, UAS-CD4-tdGFP/UAS-Kir 2.1 (experiment); $n=20$ to 22, average of 2 independent biological replicates. (f) Sample images of control and (g) Kir2.1 expressing larva. Note that Kir2.1 coexpression reduces GFP expression in PNS and CNS; CNS on left side (anterior). (h) Comparison of hemocyte expansion in $A c t \beta^{E D 80}$ mutant larvae and controls, genotypes $\mathrm{Hml} \Delta$-DsRed/ +; Act $\beta$ ED80/ED80 and control Hml $\Delta$-DsRed/ + . Total hemocyte counts per larva of carbachol-treated cohorts $\left(0.7 \mathrm{mg} \mathrm{ml}^{-1}\right)$ and control food cohorts; $n=6-8$ per genotype and condition. (i,j) Comparison of control and $A c t \beta^{E D 80}$ mutant in the recruitment of hemocytes to HPs after short-term carbachol exposure $\left(10 \mathrm{mg} \mathrm{ml}^{-1}, 15 \mathrm{~min}\right)$, genotypes as in $\mathbf{h}$, dorsal view. (k) Model. Act $\beta$ in sensory neuron-induced blood cell adaptation. PNS neuronal activity triggered by carbachol elevates Act $\beta$ expression in neurons, resulting in enhanced hemocyte proliferation and localization to HP. Neuronal silencing has opposite effects. Scale bars, $\mathbf{c}, \mathbf{d}, 100 \mu \mathrm{m} ; \mathbf{f}, \mathbf{g}, 0.5 \mathrm{~mm} ; \mathbf{i}, \mathbf{j}, 0.2 \mathrm{~mm}$. Error bars represent s.d., and two-tailed $t$-test values correspond to NS (not significant) $P>0.05 ;{ }^{\star} P \leq 0.05 ;{ }^{\star \star} P \leq 0.01 ;{ }^{\star \star \star} P \leq 0.001 ;{ }^{\star \star \star \star} P \leq 0.0001$.
} 
or near Salm, Bloomington BN46802); 21-7-GAL4 (refs 4,41), repo-GAL4 (ref. 29), He-GAL4 (ref. 61), OK6-GAL4 (Bloomington BN64199 (ref. 62)). The line Hml 4 -Gal4, UAS-GFP; He-GAL4 was a kind gift of Jesper Kronhamn and Dan Hultmark. UAS lines used were UAS-CD4-td GFP (ref. 63), UAS-mCD8-GFP (ref. 64), UAS-Stinger (Bloomington), UAS-luciferase (gift from Katherine Sepp), UAS-Act $\beta$ (ref. 19), UAS-babo(CA) (ref. ${ }^{11}$ ), UAS-Kir2.1 (ref. 27), UAS-shi dn ${ }^{\text {ts }}$ (ref. 28). UAS-RNAi lines for $d S m a d 2$, punt, babo, Act- $\beta$ and $t k v$ from TRIP, VDRC and ${ }^{19,65}$, in some cases in combination (for example, for Act $\beta$ silencing UAS-Act $\beta$ RNAi 4A2; UAS-Act $\beta$ RNAi VDRC ID 12174 was used; for $d S m a d 2$ silencing UAS-dSmad2 RNAi 3A2; UAS-Smox RNAi VDRC was used). Control crosses used yw (Bloomington) or $w^{1118}$ (Bloomington) as indicated. Other lines used were split GFP Drosophila lines from Kristin Scott ${ }^{9}$. Repo-LexA::GAD from Tzumin Lee ${ }^{66}$; Cha3.3kb-LexA::GAD from Soeren Dieglemann and Matthias Landgraf ${ }^{67}$. Unless noted otherwise, fly crosses were maintained at $25^{\circ} \mathrm{C}$, for in vivo $\mathrm{RNAi}$ at $27^{\circ} \mathrm{C}$.

Immunohistochemistry and microscopy. Antibodies used were anti-HRP-FITC (1:500, Jackson) for pan-neuronal staining ${ }^{68}$, mouse or rat anti-elav (1:50, Developmental Studies Hybridoma Bank), rabbit anti-PS4 (1:100, M. Crozatier) for lamellocytes, mouse anti-Lz (1:10, Developmental Studies Hybridoma Bank) for crystal cells, goat anti-GFP (1:2,000, Rockland), rabbit anti-RFP (1:1,000, Rockland), and Alexa fluorophore conjugated secondary antibodies (1:200, Molecular Probes). Hemocytes were released by dissecting larvae in a drop of Schneider's medium (Gibco, Invitrogen) or PBS. To bleed circulating hemocytes, larvae were opened at the ventral anterior and posterior sides, and hemolymph was allowed to leak out, avoiding to apply pressure. To prevent dislodging of resident hemocytes or contamination with lymph gland hemocytes, larvae were monitored through a fluorescence stereomicroscope. Resident hemocytes were released by opening the remainder of the larva and scraping the body wall with a needle under microscope guidance, avoiding the lymph gland. For the release of total hemocyte numbers, both procedures were combined. Hemocytes were allowed to attach to glass slides for $15-30 \mathrm{~min}$, followed by standard fixation (4\% PFA) and immunocytochemistry ${ }^{4,5}$. For larval fillet preps, larvae were pinned down on Sylgard plates and ventrally filleted in a drop of PBS. Gut, fat body and trachea were removed, and the fillet was fixed for $20 \mathrm{~min}$ in $4 \%$ PFA. Fillets were washed in PBS, unpinned, permeabilized, blocked and immunostained according to standard protocols, staining overnight with gentle agitation ${ }^{4,5}$. Lymph gland dissections and stainings were performed as described in (ref. 69), using sets of larvae 72-80 h AEL $(2.5-2.8 \mathrm{~mm})$. Images were obtained on a Leica DMI4000B, and live larvae and larval fillets were imaged on a Leica M205FA stereomicroscope ${ }^{4}$. Leica SP5 confocal microscopy was used to obtain high-resolution images. In all experiments, identical settings for experiments and controls were maintained, and images were processed in Adobe Photoshop using identical recorded action settings.

Hemocyte quantification. To quantify resident hemocytes in segmental HPs, blood cells were visualized by fluorescence microscopy and counted by external inspection of intact live larvae, excluding the compact clusters of hemocytes in the terminal segment HP. Total numbers of hemocytes per larva, and percentages of circulating hemocytes were determined by selective release and automated hemocyte quantification using Image $]^{22}$. 'Total Hemocytes' = [number of circulating hemocytes] + [number of resident hemocytes]. "\% Circulating Hemocytes' $^{\prime}=[$ number of circulating hemocytes $] \times 100 /$ [total hemocytes]. Unless indicated otherwise, experiments used larvae 72-80 h AEL (2.5-2.8 mm length), that is, late 2 nd instar larvae 4 . This was achieved by timed embryo collections and measuring larval size using Leica LAS software. For total hemocyte counts, hemocyte numbers of experiment larvae were normalized relative to backgroundmatched parallel controls. Depending on the individual experiment, baseline counts of matched controls were typically in the range of 2,000 or 3,000 hemocytes; an example of non-normalized counts over a time course can be seen in Supplementary Fig. 2. Hemocyte number averages and circulating hemocyte fraction averages from at least 4-6 larvae per genotype were calculated. The number of larvae $\mathrm{n}$ per genotype is listed in the figure legends, with lower numbers typically referring to the control. Phenotypes were confirmed in independent biological replicates. Where applicable, standard deviations and 2-tailed $t$-tests were calculated. $T$-test values correspond to the following: NS (not significant) $P>0.05 ;{ }^{*} P \leq 0.05 ;{ }^{* *} P \leq 0.01 ;{ }^{* * *} P \leq 0.001 ;{ }^{* * * *} P \leq 0.0001$.

Hemocyte re-adhesion assay. We used an in vivo hemocyte re-adhesion assay ${ }^{22}$. Specifically, hemocytes were dislodged by paint brush manipulation, and the fraction of hemocytes not returning to the resident state was determined after $45 \mathrm{~min}$ incubation, using quantitative isolation of circulating and resident hemocyte populations from single larvae $e^{22}$.

Apoptosis and cell proliferation assays. EdU (5-ethynyl-2'-deoxyuridine, Invitrogen) was used at $10 \mathrm{mM}$ for $2 \mathrm{~h}$ for in vivo feeding experiments, or $1 \mathrm{mM}$ for $2 \mathrm{~h}$ in cell culture medium. Hemocytes were released by scraping the larval carcasses, and stained in multi-well cell culture dishes ex vivo ${ }^{4,22}$. Click-iT EdU proliferation assays (Invitrogen), and TUNEL assays to quantify apoptotic cells (In Situ Cell Death Detection Kit, Roche Diagnostics) were performed according to manufacturers' instructions and used in hemocyte experiments previously ${ }^{4}$. For hemocyte ex vivo Act $\beta$ stimulation, Act $\beta$ was generated by transiently transfecting (Effectene, Qiagen) the plasmid $p A c P A-d A c t \beta^{70}$ and a control plasmid ( $p A c t 5 C$ GAL4 (M.Zeidler)) each into two Drosophila cells lines, changing medium after $8 \mathrm{~h}$, and collecting conditioned media $48 \mathrm{~h}$ after the previous media change. Hemocytes of Hml - -GAL4, UAS-GFP; He-GAL4 larvae (72-78 h AEL, corresponding to 2.5-2.7 mm) were released in S2 medium (Gibco/Invitrogen). After settling of the hemocytes $(\sim 15 \mathrm{~min})$, medium was replaced by the Act $\beta$ conditioned media or control media. Starting $1 \mathrm{~h}$ after stimulation, EdU was added for $2 \mathrm{~h}$, and cells were fixed and stained as described above. Fractions of EdU positive cells among GFP positive hemocytes where quantified by Metamorph, maintaining identical settings between stimulated and unstimulated conditions. For in vivo EdU experiments, EdU positive hemocytes were quantified by Metamorph and/or manual counting.

Manipulation of neuronal activity. Genotypes for neuronal silencing experiments were 21-7-GAL4, UAS-mCD8GFP, HmlA-DsRed/UAS-Kir2.1; tubGAL80 ${ }^{t_{s}} /+$, with controls 21-7-GAL4, UAS-mCD8GFP, Hml4-DsRed/ +. Endocytosis and synaptic vesicle recycling were blocked by temperature-sensitive dominant-negative shibire in peripheral md neurons (21-7 GAL4, UAS-mCD8GFP, Hml L-DsRed/ + ; UAS-shi $d n^{t s} /+$ ) with control larvae (21-7 GAL4, UAS-mCD8GFP, HmlL-DsRed/ +). For short term, maximal hyperpolarization, Kir2.1 or shi $d n$ ts expression was reversibly induced at $37^{\circ} \mathrm{C}(\mathrm{Kir} 2.1)$ or $34^{\circ} \mathrm{C}$ (shi dn ts) for $30 \mathrm{~min}$ and larvae were analysed within an hour. For long term, moderate silencing, larvae were induced at $27^{\circ} \mathrm{C}$ for the indicated periods of time from larval hatching onward. For expression of Kir2.1 and shi dn ts in glia as a control tissue, HmlA-DsRed; repo-GAL4, UAS$m C D 8 G F P$ line was crossed to UAS-Kir2.1; tubGAL80 ${ }^{\text {ts }}$ or UAS-shi $d n^{\text {ts }}$ respectively. Larvae of the genotype HmlL-DsRed/ + ; repo-GAL4, UAS-mCD8GFP/+, which did not express the silencing proteins, were included as additional controls For maximal effects, larvae were induced at $37^{\circ} \mathrm{C}$ for $30 \mathrm{~min}$ and analysed within an hour after. For all experiments, embryo collections were timed to $12 \mathrm{~h}$ or less and embryogenesis was completed at $18{ }^{\circ} \mathrm{C}$ before inducing neuronal silencing. Intact larvae were exposed to carbamoylcholine (carbachol), added to water to expose larvae via the cuticle surface in short-term stimulations (at 2.5 or $10 \mathrm{mg} \mathrm{ml}^{-1}$ ), or added to fly food for long-term experiments (at $0.7 \mathrm{mg} \mathrm{ml}^{-1}$ ).

Act $\beta$-GAL4 reporter luciferase assay and quantification of GFP expression. Act $\beta$ transcription was quantified based on combining UAS-luciferase and/or UAS$C D 4-t d G F P$ with the Activin- $\beta$ enhancer reporter Act $\beta$-GAL4. Larvae were stimulated with carbachol for $2-3 \mathrm{~h}$ (see above). Before lysis, larvae were dissected and the anterior portion with the highly Act $\beta$-GAL4 expressing CNS was carefully removed before lysis. Luciferase levels were quantified using Bright-Glo according to the manufacturer's instructions (Promega). For quantification of GFP expression, images of controls and carbachol-stimulated fillets were taken at identical settings (Leica M205 fluorescence stereoscope or Leica SP5 confocal). Images were cropped to corresponding regions of identical areas, and image signal intensity was quantified using ImageJ. Signal intensity of carbachol-stimulated samples relative to unstimulated controls was calculated and averaged.

GFP reconstitution across synaptic partners (GRASP). The following Drosophila genetic combinations were generated and crossed to detect contact between sensory neurons and hemocytes: lexAop-CD2::GFP11(11-3)/CyO;Cha3-LexAGAD\#4/Tb and UAS-CD4::spGFP1-10(4.1), HmlL-DsRed/CyO; Pxn-GAL4/Tb. To observe contacts between glia and hemocytes the following genetic combinations were generated and crossed: repo-LexA-GAD; lexAop-CD4::spGFP11(11-3)/CyO, P\{Wee-P. ph0\}2 and UAS-CD4::spGFP1-10(4.1), HmlL-DsRed/CyO; Pxn-GAL4/Tb. Larvae were filleted and imaged using confocal microscopy.

Data availability. The authors declare that all data supporting the findings of this study are available within the article and its Supplementary Information files or from the corresponding author on reasonable request.

\section{References}

1. Davies, L. C., Jenkins, S. J., Allen, J. E. \& Taylor, P. R. Tissue-resident macrophages. Nat. Immunol. 14, 986-995 (2013).

2. Sieweke, M. H. \& Allen, J. E. Beyond stem cells: self-renewal of differentiated macrophages. Science 342, 1242974 (2013).

3. Gomez Perdiguero, E. et al. Tissue-resident macrophages originate from yolksac-derived erythro-myeloid progenitors. Nature 518, 547-551 (2015).

4. Makhijani, K., Alexander, B., Tanaka, T., Rulifson, E. \& Brückner, K. The peripheral nervous system supports blood cell homing and survival in the Drosophila larva. Development 138, 5379-5391 (2011).

5. Makhijani, K. \& Brückner, K. Of blood cells and the nervous system: Hematopoiesis in the Drosophila larva. Fly 6, 254-260 (2012).

6. Gold, K. S. \& Brückner, K. Drosophila as a model for the two myeloid blood cell systems in vertebrates. Exp. Hematol. 42, 717-727 (2014).

7. Gold, K. S. \& Brückner, K. Macrophages and cellular immunity in Drosophila melanogaster. Semin. Immunol. 27, 357-368 (2015). 
8. Feinberg, E. H. et al. GFP reconstitution across synaptic partners (GRASP) defines cell contacts and synapses in living nervous systems. Neuron 57, 353-363 (2008).

9. Gordon, M. D. \& Scott, K. Motor control in a Drosophila taste circuit. Neuron 61, 373-384 (2009).

10. Dietzl, G. et al. A genome-wide transgenic RNAi library for conditional gene inactivation in Drosophila. Nature 448, 151-156 (2007).

11. Brummel, T. et al. The Drosophila activin receptor baboon signals through $\mathrm{dSmad} 2$ and controls cell proliferation but not patterning during larval development. Genes Dev. 13, 98-111 (1999).

12. Clark, R. I., Woodcock, K. J., Geissmann, F., Trouillet, C. \& Dionne, M. S. Multiple TGF-beta superfamily signals modulate the adult Drosophila immune response. Curr. Biol. 21, 1672-1677 (2011).

13. Awasaki, T., Huang, Y., O'Connor, M. B. \& Lee, T. Glia instruct developmental neuronal remodeling through TGF-beta signaling. Nat. Neurosci. 14, 821-823 (2011).

14. Orgogozo, V. \& Grueber, W. B. FlyPNS, a database of the Drosophila embryonic and larval peripheral nervous system. BMC Dev. Biol. 5, 4 (2005).

15. Zheng, X. et al. TGF-beta signaling activates steroid hormone receptor expression during neuronal remodeling in the Drosophila brain. Cell 112, 303-315 (2003)

16. Serpe, M. \& O'Connor, M. B. The metalloprotease tolloid-related and its TGF-beta-like substrate Dawdle regulate Drosophila motoneuron axon guidance. Development 133, 4969-4979 (2006).

17. Parker, L., Ellis, J. E., Nguyen, M. Q. \& Arora, K. The divergent TGF-beta ligand Dawdle utilizes an activin pathway to influence axon guidance in Drosophila. Development 133, 4981-4991 (2006).

18. Ting, C. Y. et al. Tiling of $\mathrm{r} 7$ axons in the Drosophila visual system is mediated both by transduction of an activin signal to the nucleus and by mutual repulsion. Neuron 56, 793-806 (2007).

19. Gibbens, Y. Y., Warren, J. T., Gilbert, L. I. \& O'Connor, M. B. Neuroendocrine regulation of Drosophila metamorphosis requires TGFbeta/Activin signaling. Development 138, 2693-2703 (2011)

20. Kim, M. J. \& O'Connor, M. B. Anterograde activin signaling regulates postsynaptic membrane potential and GluRIIA/B abundance at the Drosophila neuromuscular junction. PLoS ONE 9, e107443 (2014)

21. Zhu, C. C. et al. Drosophila activin- and the activin-like product Dawdle function redundantly to regulate proliferation in the larval brain. Development 135, 513-521 (2008).

22. Petraki, S., Alexander, B. \& Brückner, K. Assaying blood cell populations of the Drosophila melanogaster larva. J. Vis. Exp. 105, e52733 (2015).

23. Letsou, A. et al. Drosophila Dpp signaling is mediated by the punt gene product: a dual ligand-binding type II receptor of the TGF beta receptor family. Cell 80, 899-908 (1995).

24. Bretscher, A. J. et al. The Nimrod transmembrane receptor Eater is required for hemocyte attachment to the sessile compartment in Drosophila melanogaster. Biol. Open 4, 355-363 (2015).

25. Leitao, A. B. \& Sucena, E. Drosophila sessile hemocyte clusters are true hematopoietic tissues that regulate larval blood cell differentiation. Elife 4, e06166 (2015).

26. Salvaterra, P. M. \& Kitamoto, T. Drosophila cholinergic neurons and processes visualized with Gal4/UAS-GFP. Brain Res. Gene Exp. Patterns 1, 7382 (2001).

27. Baines, R. A., Uhler, J. P., Thompson, A., Sweeney, S. T. \& Bate, M. Altered electrical properties in Drosophila neurons developing without synaptic transmission. J. Neurosci. 21, 1523-1531 (2001).

28. Kitamoto, T. Targeted expression of temperature-sensitive dynamin to study neural mechanisms of complex behavior in Drosophila. J. Neurogenet. 16, 205-228 (2002)

29. Sepp, K. J. \& Auld, V. J. Conversion of lacZ enhancer trap lines to GAL4 lines using targeted transposition in Drosophila melanogaster. Genetics 151, 1093-1101 (1999).

30. Xiang, Y. et al. Light-avoidance-mediating photoreceptors tile the Drosophila larval body wall. Nature 468, 921-926 (2010).

31. Im, S. H. \& Galko, M. J. Pokes, sunburn, and hot sauce: Drosophila as an emerging model for the biology of nociception. Dev. Dyn. 241, 16-26 (2012).

32. Morikawa, M., Derynck, R. \& Miyazono, K. TGF-beta and the TGF-beta family: context-dependent roles in cell and tissue physiology. Cold Spring Harb. Perspect. Biol. 8, a021873 (2016).

33. Zhang, Y., Alexander, P. B. \& Wang, X. F. TGF-beta family signaling in the control of cell proliferation and survival. Cold Spring Harb. Perspect. Biol. 9, a022145 (2017).

34. Yang, S. A. \& Su, M. T. Excessive Dpp signaling induces cardial apoptosis through dTAK1 and dJNK during late embryogenesis of Drosophila. J. Biomed. Sci. 18, 85 (2011)

35. Lengil, T., Gancz, D. \& Gilboa, L. Activin signaling balances proliferation and differentiation of ovarian niche precursors and enables adjustment of niche numbers. Development 142, 883-892 (2015).
36. Ghosh, A. C. \& O'Connor, M. B. Systemic Activin signaling independently regulates sugar homeostasis, cellular metabolism, and $\mathrm{pH}$ balance in Drosophila melanogaster. Proc. Natl Acad. Sci. USA 111, 5729-5734 (2014).

37. Serpe, M., Ralston, A., Blair, S. S. \& O’Connor, M. B. Matching catalytic activity to developmental function: tolloid-related processes Sog in order to help specify the posterior crossvein in the Drosophila wing. Development 132, 2645-2656 (2005).

38. Peterson, A. J. \& O'Connor, M. B. Activin receptor inhibition by Smad2 regulates Drosophila wing disc patterning through BMP-response elements. Development 140, 649-659 (2013).

39. Akiyama, T., Marques, G. \& Wharton, K. A. A large bioactive BMP ligand with distinct signaling properties is produced by alternative proconvertase processing. Sci. Signal. 5, ra28 (2012).

40. Constam, D. B. Regulation of TGFbeta and related signals by precursor processing. Semin. Cell Dev. Biol. 32, 85-97 (2014).

41. Song, W., Onishi, M., Jan, L. Y. \& Jan, Y. N. Peripheral multidendritic sensory neurons are necessary for rhythmic locomotion behavior in Drosophila larvae. Proc. Natl Acad. Sci. USA 104, 5199-5204 (2007).

42. Christiansen, F. et al. Presynapses in Kenyon cell dendrites in the mushroom body calyx of Drosophila. J. Neurosci. 31, 9696-9707 (2011).

43. Bergquist, F. \& Ludwig, M. Dendritic transmitter release: a comparison of two model systems. J. Neuroendocrinol. 20, 677-686 (2008).

44. Miyashita, T. et al. $\mathrm{Mg}^{2}+$ block of Drosophila NMDA receptors is required for long-term memory formation and CREB-dependent gene expression. Neuron 74, 887-898 (2012).

45. Inokuchi, K. et al. Increase in activin beta A mRNA in rat hippocampus during long-term potentiation. FEBS Lett. 382, 48-52 (1996).

46. Dahal, G. R. et al. An inwardly rectifying $\mathrm{K}+$ channel is required for patterning. Development 139, 3653-3664 (2012).

47. Herbomel, P., Thisse, B. \& Thisse, C. Zebrafish early macrophages colonize cephalic mesenchyme and developing brain, retina, and epidermis through a M-CSF receptor-dependent invasive process. Dev. Biol. 238, 274-288 (2001).

48. Schulz, C. et al. A lineage of myeloid cells independent of Myb and hematopoietic stem cells. Science 336, 86-90 (2012).

49. Sozzani, S. \& Musso, T. The yin and yang of Activin A. Blood 117, 5013-5015 (2011)

50. Hussell, T. \& Bell, T. J. Alveolar macrophages: plasticity in a tissue-specific context. Nat. Rev. Immunol. 14, 81-93 (2014).

51. Katayama, Y. et al. Signals from the sympathetic nervous system regulate hematopoietic stem cell egress from bone marrow. Cell 124, 407-421 (2006).

52. Mendez-Ferrer, S., Lucas, D., Battista, M. \& Frenette, P. S. Haematopoietic stem cell release is regulated by circadian oscillations. Nature 452, 442-447 (2008)

53. Spiegel, A. et al. Catecholaminergic neurotransmitters regulate migration and repopulation of immature human $\mathrm{CD} 34+$ cells through Wnt signaling. Nat. Immunol. 8, 1123-1131 (2007).

54. Yamazaki, S. et al. Nonmyelinating schwann cells maintain hematopoietic stem cell hibernation in the bone marrow niche. Cell 147, 1146-1158 (2011).

55. Brückner, K. Blood cells need glia, too: a new role for the nervous system in the bone marrow niche. Cell Stem Cell 9, 493-495 (2011).

56. Boulais, N. \& Misery, L. The epidermis: a sensory tissue. Eur. J. Dermatol. 18, 119-127 (2008).

57. Chang, R. B., Strochlic, D. E., Williams, E. K., Umans, B. D. \& Liberles, S. D. Vagal sensory neuron subtypes that differentially control breathing. Cell 161, 622-633 (2015).

58. Pauziene, N. et al. Innervation of the rabbit cardiac ventricles. J. Anat. 228, 26-46 (2016).

59. Razavi, R. et al. TRPV1 + sensory neurons control beta cell stress and islet inflammation in autoimmune diabetes. Cell 127, 1123-1135 (2006).

60. McGuire, S. E., Le, P. T., Osborn, A. J., Matsumoto, K. \& Davis, R. L. Spatiotemporal rescue of memory dysfunction in Drosophila. Science 302, 1765-1768 (2003).

61. Zettervall, C. J. et al. A directed screen for genes involved in Drosophila blood cell activation. Proc. Natl Acad. Sci. USA 101, 14192-14197 (2004).

62. Aberle, H. et al. wishful thinking encodes a BMP type II receptor that regulates synaptic growth in Drosophila. Neuron 33, 545-558 (2002).

63. Han, C., Jan, L. Y. \& Jan, Y. N. Enhancer-driven membrane markers for analysis of nonautonomous mechanisms reveal neuron-glia interactions in Drosophila. Proc. Natl Acad. Sci. USA 108, 9673-9678 (2011).

64. Lee, T. \& Luo, L. Mosaic analysis with a repressible cell marker for studies of gene function in neuronal morphogenesis. Neuron 22, 451-461 (1999).

65. Peterson, A. J. et al. R-smad competition controls activin receptor output in Drosophila. PLoS ONE 7, e36548 (2012).

66. Lai, S. L. \& Lee, T. Genetic mosaic with dual binary transcriptional systems in Drosophila. Nat. Neurosci. 9, 703-709 (2006).

67. Diegelmann, S., Bate, M. \& Landgraf, M. Gateway cloning vectors for the LexAbased binary expression system in Drosophila. Fly 2, 236-239 (2008)

68. Jan, L. Y. \& Jan, Y. N. Antibodies to horseradish peroxidase as specific neuronal markers in Drosophila and in grasshopper embryos. Proc. Natl Acad. Sci. USA 79, 2700-2704 (1982). 
69. Evans, C. J., Liu, T. \& Banerjee, U. Drosophila hematopoiesis: markers and methods for molecular genetic analysis. Methods 68, 242-251 (2014).

70. Gesualdi, S. C. \& Haerry, T. E. Distinct signaling of Drosophila Activin/TGFbeta family members. Fly 1, 212-221 (2007).

\section{Acknowledgements}

We thank M. Crozatier, S. Diegelmann, C. Evans, C. Han, U. Heberlein, Y.N. Jan, L. Kockel, M. Landgraf, T. Lee, L. Luo, B. Mathey-Prevot, P. Rao, J. Schulte, K. Scott, O. Shafer, K. Sepp, S. Sinenko, S. Younger, M. Zeidler, the Bloomington Stock Center, and the DGRC for antibodies, stocks, plasmids, or sequence information. Special thanks to J. Kronhamn and D. Hultmark for the line HmlA-Gal4, UAS-GFP; He-GAL4. K.M. was supported by a HFSP long-term fellowship. K.S.G. was supported by an American Heart Association fellowship. This work was supported by grants from the UCSF Program for Breakthrough Biomedical Research (PBBR), Broad Center, Hellman Foundation, American Cancer Society RSG DDC-122595, American Heart Association 13BGIA13730001, National Science Foundation 1326268, National Institutes of Health 1R01GM112083-01 and 1R56HL118726-01A1 (to K.B.). This investigation was in part conducted in a facility constructed with support from the Research Facilities Improvement Program, Grant number C06-RR16490 from the NCRR/NIH.

\section{Author contributions}

K.B. and K.M. designed experiments. K.M., B.A., D.R., S.P., L.H., K.K., I.B., S.W., K.S.G., C.W. and K.B. performed experiments. M.B.O. provided Activin- $\beta$ pathway tools and expertise. K.B. conceived the study. K.B., K.M., B.A., D.R., S.P., L.H., K.K., I.B., S.W. and K.S.G. evaluated the data and K.B. wrote the manuscript with input from all authors.

\section{Additional information}

Supplementary Information accompanies this paper at http://www.nature.com/ naturecommunications

Competing interests: The authors declare no competing financial interests.

Reprints and permission information is available online at http://npg.nature.com/ reprintsandpermissions/

How to cite this article: Makhijani, K. et al. Regulation of Drosophila hematopoietic sites by Activin- $\beta$ from active sensory neurons. Nat. Commun. 8, 15990 doi: $10.1038 /$ ncomms15990 (2017).

Publisher's note: Springer Nature remains neutral with regard to jurisdictional claims in published maps and institutional affiliations.

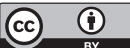

Open Access This article is licensed under a Creative Commons Attribution 4.0 International License, which permits use, sharing, adaptation, distribution and reproduction in any medium or format, as long as you give appropriate credit to the original author(s) and the source, provide a link to the Creative Commons license, and indicate if changes were made. The images or other third party material in this article are included in the article's Creative Commons license, unless indicated otherwise in a credit line to the material. If material is not included in the article's Creative Commons license and your intended use is not permitted by statutory regulation or exceeds the permitted use, you will need to obtain permission directly from the copyright holder. To view a copy of this license, visit http://creativecommons.org/ licenses/by/4.0/

(C) The Author(s) 2017 